\title{
Cross-Sectional Returns and Fama-MacBeth Betas for S\&P Indices
}

\author{
V. Reddy Dondeti ${ }^{1} \&$ Carl B. McGowan, Jr. ${ }^{1}$ \\ ${ }^{1}$ School of Business, Norfolk State University, Norfolk, VA 23504, USA \\ Correspondence: Carl B. McGowan, Jr., School of Business, Norfolk State University, Norfolk, VA 23504, USA. \\ Tel: 1-757-275-6876 E-mail: cbmcgowan@yahoo.com
}

Received: October 26, 2013

Accepted: November 19, $2013 \quad$ Online Published: November 20, 2013

doi:10.5430/afr.v2n4p149

URL: http://dx.doi.org/10.5430/afr.v2n4p149

\begin{abstract}
In this paper, we use the Fama-MacBeth regression analysis methodology to determine if twenty indices for the twenty year time period from 1990 to 2009 provide a linear relationship between the index returns and index betas. The time-series of the betas of all the indices except that of Gold and Silver Index for monthly returns of one-year intervals are non-stationary. The betas in four of the five quintiles formed by sorting the indices in order of the highest to the lowest betas are found to be co-integrated. The results of the empirical tests on the gamma coefficients of the Fama-Macbeth regressions do not support the CAPM.
\end{abstract}

Keywords: Fama-MacBeth betas, S\&P Indices, Stationarity of betas, Co-integrated betas, Gamma coefficient analysis

\section{Introduction}

A market index like S\&P 500, Dow Jones Industrials Average or Nasdaq Composite is a portfolio constructed from a set of pre-selected stocks traded on various stock exchanges. Of course, some indices like the Dow Jones Industrials Average may have a fixed number of stocks, whereas other indices like the Nasdaq Composite or the Dow Jones Total Market may have a large number of stocks with some periodic additions and deletions. Fama and MacBeth (1973) use Fisher's Arithmetic Index, an equally weighted average of the returns of all the stocks traded on NYSE at that time, as a proxy for the market return $\tilde{R}_{m t}$. There are several popular market indices whose daily values are reported in the financial press. Also, in recent years, the S\&P 500 index has become the proxy for market return. Many financial planners advise their clients to invest their money in index-based funds. Therefore, it is reasonable to assume that individual investors would look at several market indices before they invest money in stocks, mutual funds (index-based or sector-based) or other assets, and therefore it is to be expected that they would be interested in the betas of the market indices relative to the S\&P 500 index. One of the objectives of the current study is to test whether the Capital Asset Pricing Model (CAPM) can be a valid predictor of the cross-sectional returns of some well-known market indices, using the S\&P 500 index as proxy for the market return.

Section 2 of this paper discusses the Capital Asset Pricing Model. Section 3 explains the data sample and Section 4 discusses the values of the average monthly returns for the twenty indices which include Dow Jones, NYSE, NASDAQ, S\&P, and Russell indices as well as the PHLX Gold and Silver Index. Section 5 discusses the estimates of the Fama-MacBeth betas for the twenty different indices calculated for various time periods and Section 6 discusses the unit root tests for the levels of betas with no intercept or trend of the one-year Fama-MacBeth betas. Section 7 discusses the gamma calculations and tests. Section 8 provides the conclusions of this paper.

\section{The Capital Asset Pricing Model}

In its most basic form, the Capital Asset Pricing Model (CAPM) defines the equilibrium relationship between the expected return and risk of an asset relative to a market portfolio. The equation (Fama and MacBeth, 1973) that links the expected return and risk of the asset is:

$$
E\left(\tilde{R}_{i}\right)=E\left(\tilde{R}_{0}\right)+\left[E\left(\tilde{R}_{m}\right)-E\left(\tilde{R}_{0}\right)\right] \beta_{i}
$$

Where: $\tilde{R}_{i}$ and $\tilde{R}_{m}$ are the returns on asset $i$ and the market portfolio,

$\beta_{i}=\operatorname{cov}\left(\tilde{R}_{i}, \tilde{R}_{m}\right) / \sigma_{m}^{2}$, and $\tilde{R}_{0}$ is the risk-free rate of return with $\beta=0$.

To test equation (1) empirically, it is re-stated as:

$$
\tilde{R}_{i t}=a_{i}+\beta_{i} \tilde{R}_{m t}+\tilde{\varepsilon}_{i t}
$$


If $i$ is not a single asset, but a portfolio $p$ made of assets $j, j=1,2, \ldots, J$, equations (1) and (2) still hold good, and in empirical testable form, equation (2) is written as:

$$
R_{p t}=\hat{\gamma}_{0 t}+\hat{\gamma}_{1 t} \hat{\beta}_{p, t-1}+\hat{\gamma}_{2 t} \hat{\beta}_{p, t-1}^{2}+\hat{\gamma}_{3 t} \bar{s}_{p, t-1}\left(\hat{\varepsilon}_{i}\right)+\hat{\eta}_{p t}
$$

The procedures for constructing a finite number (20 to 100) of different portfolios from hundreds of stocks traded in different markets are described in Fama and MacBeth (1973), Fama and French (1992, 1996), and Jagannathan and Wang (1996). However, in the current study, no portfolios are constructed. A market index replaces the constructed portfolio $p$.

\section{Data Sample}

The data for this study is collected from the Global Financial Database (GFD). The market indices that will be included in the study are given in Table 1.

Table 1. Market Indices

\begin{tabular}{|c|c|c|c|}
\hline Series ID & Name of Market Index & Symbol in GFD & Dates of data availability \\
\hline R00_SP500 & S\&P 500 & SPXD & $1791-2009$ \\
\hline R01_DJIA & Dow Jones Industrials Average & DWI_XD & $1896-2009$ \\
\hline R02_DJTran & Dow Jones Transportation Average & DWT_XD & $1889-2009$ \\
\hline R03_DJUtil & Dow Jones Utility Average & DWU_D & $1929-2009$ \\
\hline R04_DJTotal & Dow Jones Total U.S. market & DWCD & $1970-2009$ \\
\hline R05_NYComp & NYSE Composite (New) & NYAD & $1929-2009$ \\
\hline R06_NDComp & NASDAQ Composite & OTC_D & $1938-2009$ \\
\hline R07_ND100 & NASDAQ 100 & NDXD & $1985-2009$ \\
\hline R08_NDBank & NASDAQ Bank Index & IXBKD & 1971-2009 \\
\hline R09_NDIns & NASDAQ Insurance & IXISD & $1965-2009$ \\
\hline R10_NDTel & NASDAQ Telecommunications & IXUTD & $1971-2009$ \\
\hline R11_SP100 & S\&P 100 & OEXD & $1976-2009$ \\
\hline R12_SP400 & S\&P 400 Mid-cap & IDXD & $1981-2009$ \\
\hline R13_SP600 & S\&P 600 Small-Cap Index & SMLD & 1989-2009 \\
\hline R14_SPAero & S\&P 500 Aerospace \& Defense & GSPAEDD & $1928-2009$ \\
\hline R15_SPMatl & S\&P 500 Materials & GSPMD & 1989-2009 \\
\hline R16_SPInfo & S\&P 500 Information Technology & GSPTD & 1986-2009 \\
\hline R17_SPHeal & S\&P Healthcare Composite & HCXD & $1987-2009$ \\
\hline R18_RS1000 & Russell 1000 Index & RUID & $1978-2009$ \\
\hline R19_RS2000 & Russell 2000 Index & RUTD & $1978-2009$ \\
\hline R20_GoldSil & PHLX Gold and Silver Index & XAUD & $1983-2009$ \\
\hline
\end{tabular}

For two indices, S\&P 600 Small Cap Index and S\&P 500 Materials, data is available only from the middle of 1989 onwards. Therefore, the analysis will cover the years from 1990 to 2009 (a time span of 20 years). The selected indices cover a broad spectrum of industries that are of interest to a variety of investors. Some investors may be interested in the Utilities index which is known to be less volatile. Others may be interested in the highly volatile Nasdaq 100 index. Some others may be interested in the Gold and Silver index which appears on the radar screens of investors in times of crises.

\section{Average Monthly Returns}

The average monthly returns of the different indices are given in Table 2. First, it may be noted that the compositions of the indices are not mutually exclusive; several of these indices contain stocks of many of the largest companies. Therefore, it is expected that the broad indices would exhibit somewhat similar pattern of returns and volatility. Several observations can be made from the results displayed in Table 2: (a) The Nasdaq 100 index (series R07) has the highest mean monthly return of $1.2 \%$ over the 20 -year period, whereas the Gold and Silver index (R20) has 
recorded the maximum and minimum monthly returns of $53.4 \%$ and $-38.2 \%$. Further, the Gold and Silver index also has the highest volatility (standard deviation of monthly returns) of $10.4 \%$. The Dow Jones Utilities index (series R03) has the lowest monthly return. Broader indices such as S\&P 500 (series R00), Dow Jones Total Market (series R04), NYSE Composite (series R05), and Russell 1000 (series R18) have comparable returns and volatility.

Table 2. Average Monthly Returns of Different Indices

\begin{tabular}{lccccc}
\hline Series & Mean & Median & Maximum & Minimum & Std. Dev. \\
\hline R00_SP500 & $0.575 \%$ & $1.013 \%$ & $11.159 \%$ & $-16.943 \%$ & $4.328 \%$ \\
R01_DJIA & $0.650 \%$ & $1.002 \%$ & $10.605 \%$ & $-15.132 \%$ & $4.292 \%$ \\
R02_DJTran & $0.708 \%$ & $1.551 \%$ & $17.455 \%$ & $-21.992 \%$ & $6.071 \%$ \\
R03_DJUtil & $0.320 \%$ & $0.825 \%$ & $11.758 \%$ & $-13.393 \%$ & $4.457 \%$ \\
R04_DJTotl & $0.605 \%$ & $1.194 \%$ & $10.715 \%$ & $-17.635 \%$ & $4.405 \%$ \\
R05_NYComp & $0.612 \%$ & $1.039 \%$ & $10.733 \%$ & $-19.537 \%$ & $4.215 \%$ \\
R06_NDComp & $0.920 \%$ & $1.707 \%$ & $21.976 \%$ & $-22.902 \%$ & $6.995 \%$ \\
R07_ND100 & $1.199 \%$ & $1.842 \%$ & $24.981 \%$ & $-26.405 \%$ & $7.888 \%$ \\
R08_NDBank & $0.718 \%$ & $1.193 \%$ & $11.028 \%$ & $-21.813 \%$ & $4.743 \%$ \\
R09_NDIns & $0.893 \%$ & $1.220 \%$ & $13.521 \%$ & $-16.009 \%$ & $4.499 \%$ \\
R10_NDTel & $0.580 \%$ & $1.356 \%$ & $23.694 \%$ & $-29.029 \%$ & $8.353 \%$ \\
R11_SP100 & $0.574 \%$ & $0.960 \%$ & $10.792 \%$ & $-14.591 \%$ & $4.411 \%$ \\
R12_SP400 & $0.923 \%$ & $1.239 \%$ & $14.753 \%$ & $-21.835 \%$ & $5.032 \%$ \\
R13_SP600 & $0.830 \%$ & $1.585 \%$ & $17.309 \%$ & $-20.242 \%$ & $5.436 \%$ \\
R14_SPAero & $0.883 \%$ & $1.199 \%$ & $15.088 \%$ & $-19.820 \%$ & $5.741 \%$ \\
R15_SPMat & $0.561 \%$ & $0.820 \%$ & $24.051 \%$ & $-22.177 \%$ & $5.801 \%$ \\
R16_SPInfo & $1.043 \%$ & $1.299 \%$ & $22.291 \%$ & $-28.014 \%$ & $7.866 \%$ \\
R17_SPHeal & $0.797 \%$ & $1.105 \%$ & $16.081 \%$ & $-12.842 \%$ & $4.789 \%$ \\
R18_RS1000 & $0.597 \%$ & $1.113 \%$ & $11.161 \%$ & $-17.503 \%$ & $4.373 \%$ \\
R19_RS2000 & $0.710 \%$ & $1.598 \%$ & $16.420 \%$ & $-20.904 \%$ & $5.630 \%$ \\
R20_GoldSil & $0.676 \%$ & $0.384 \%$ & $53.385 \%$ & $-38.221 \%$ & $10.424 \%$ \\
\hline
\end{tabular}

Often, Utilities are considered to be the safest of investments. In other words, investors lose the least in severe bear markets, if they own stocks of utilities. However, Table 2 shows that among all the indices, the S\&P Healthcare Composite index (series R17) has suffered the minimum loss of $12.84 \%$ vis-à-vis the loss of $13.4 \%$ suffered by the Dow Jones Utilities index (series R03). The average monthly return from S\&P Healthcare Composite index (series R17) is more than double that of the Utility index. These results indicate that investors looking for a safe haven may be better off with Healthcare index than the Utility index.

\section{Estimation of Betas}

In Fama and MacBeth (1973), the values of betas are calculated based on the monthly returns for four year or longer periods. In the current study, initially, betas are calculated for non-overlapping intervals of one-year, two-year, four-year, five-year, ten-year and the entire 20-year period. The one-year interval yields 20 beta values for each index, since the data covers 20 years. First, the means of the one-year betas are calculated and based on the means of these 20 one-year betas, the indices are sorted into quintiles in order of the highest to the lowest values and displayed in Table 3. By construction, the indices in the first quintile are the most volatile and the indices in the fifth quintile are the least volatile. The indices in the third quintile have a beta, close 1.00, and mimick the market portfolio. Again, the Gold-Silver index (series R20) is an outlier in the sense that its average beta is in the fifth quintile, but among all the indices, its beta has the maximum value of 3.073 , and the minimum value of -0.554 (the only index with a large negative beta). The Dow Jones Utilities Index has a negative minimum beta. 
Table 3. Summary Statistics of one-year betas

\begin{tabular}{|c|c|c|c|c|c|}
\hline Index & Mean & Median & Maximum & Minimum & Std. Dev. \\
\hline \multicolumn{6}{|c|}{ Panel A: Quintile 1} \\
\hline R10_NDTEL & 1.491 & 1.435 & 2.999 & 0.788 & 0.588 \\
\hline R07_ND100 & 1.445 & 1.451 & 2.532 & 0.301 & 0.495 \\
\hline R16_SPINFO & 1.430 & 1.422 & 2.540 & 0.153 & 0.549 \\
\hline R06_NDCOMP & 1.309 & 1.270 & 2.170 & 0.723 & 0.389 \\
\hline \multicolumn{6}{|c|}{ Panel B: Quintile 2} \\
\hline R19_RS2000 & 1.107 & 1.095 & 1.875 & 0.441 & 0.370 \\
\hline R15_SPMATL & 1.103 & 1.075 & 1.860 & 0.421 & 0.331 \\
\hline R02_DJTRANS & 1.083 & 1.053 & 1.946 & 0.416 & 0.352 \\
\hline R12_SP400 & 1.080 & 1.051 & 1.436 & 0.799 & 0.195 \\
\hline \multicolumn{6}{|c|}{ Panel C: Quintile 3} \\
\hline R13_SP600 & 1.077 & 1.114 & 1.647 & 0.559 & 0.319 \\
\hline R04_DJTOTAL & 1.009 & 1.001 & 1.129 & 0.916 & 0.065 \\
\hline R18_RS1000 & 1.006 & 1.004 & 1.048 & 0.973 & 0.023 \\
\hline R11_SP100 & 0.992 & 0.983 & 1.143 & 0.851 & 0.084 \\
\hline \multicolumn{6}{|c|}{ Panel D: Quintile 4} \\
\hline R01_DJIA & 0.962 & 0.952 & 1.489 & 0.739 & 0.167 \\
\hline R05_NYCOMP & 0.961 & 0.967 & 1.129 & 0.763 & 0.105 \\
\hline R14_SPAERO & 0.873 & 0.787 & 1.923 & 0.160 & 0.436 \\
\hline R17_SPHEALTH & 0.757 & 0.729 & 1.419 & 0.013 & 0.362 \\
\hline \multicolumn{6}{|c|}{ Panel E: Quintile 5} \\
\hline R20_GOLDSIL & 0.738 & 0.469 & 3.073 & -0.554 & 0.930 \\
\hline R09_NDINSR & 0.696 & 0.737 & 1.248 & 0.017 & 0.281 \\
\hline R08_NDBANK & 0.639 & 0.642 & 1.247 & 0.095 & 0.272 \\
\hline R03_DJUTIL & 0.461 & 0.475 & 1.076 & -0.093 & 0.336 \\
\hline
\end{tabular}

Table 4-A. Betas of Different Indices- Nonoverlapping Time Intervals

Panel A : Values of one-year Betas

(sorted: highest to lowest based on one-year mean beta values)

\begin{tabular}{ccccccccccc}
\hline & R10 & R07 & R16 & R06 & R19 & R15 & R02 & R12 & R13 & R04 \\
Year & NDTel & ND100 & SpInfo & NDcomp & RS2000 & SPMatl & DJTrans & SP400 & SP600 & DJTotl \\
1990 & 1.47 & 1.56 & 1.13 & 1.30 & 1.23 & 1.12 & 1.25 & 1.22 & 1.19 & 1.03 \\
1991 & 1.00 & 1.41 & 0.97 & 1.12 & 0.98 & 0.95 & 1.44 & 1.07 & 0.94 & 0.98 \\
1992 & 1.00 & 1.04 & 0.97 & 0.80 & 0.70 & 0.83 & 1.07 & 0.99 & 0.70 & 0.94 \\
1993 & 2.50 & 1.68 & 1.87 & 1.44 & 1.12 & 0.42 & 0.42 & 1.12 & 1.33 & 1.04 \\
1994 & 1.10 & 1.13 & 1.23 & 0.95 & 0.87 & 1.28 & 1.29 & 0.95 & 0.94 & 0.95 \\
1995 & 1.40 & 0.30 & 0.15 & 0.72 & 1.07 & 1.86 & 1.31 & 1.06 & 1.22 & 0.96 \\
1996 & 0.79 & 1.46 & 1.70 & 1.15 & 0.79 & 0.77 & 0.91 & 0.86 & 0.82 & 0.95 \\
1997 & 0.90 & 1.45 & 1.57 & 1.07 & 0.61 & 0.92 & 0.78 & 0.80 & 0.66 & 0.92 \\
1998 & 1.57 & 1.35 & 1.25 & 1.37 & 1.23 & 0.80 & 0.93 & 1.29 & 1.23 & 1.06 \\
1999 & 1.81 & 1.92 & 1.68 & 1.78 & 0.94 & 1.17 & 0.89 & 0.89 & 0.74 & 1.06 \\
2000 & 1.27 & 1.58 & 1.77 & 1.37 & 0.44 & 0.91 & 0.85 & 0.91 & 0.56 & 0.94 \\
2001 & 2.24 & 2.53 & 2.54 & 2.17 & 1.05 & 0.84 & 1.21 & 1.08 & 0.99 & 1.05 \\
2002 & 1.79 & 1.66 & 1.99 & 1.37 & 0.88 & 1.03 & 0.69 & 0.81 & 0.73 & 0.95 \\
2003 & 0.89 & 0.93 & 0.96 & 0.97 & 1.22 & 1.43 & 1.46 & 1.01 & 1.14 & 1.00 \\
2004 & 1.97 & 1.68 & 1.77 & 1.79 & 1.76 & 1.15 & 1.04 & 1.32 & 1.51 & 1.12 \\
2005 & 1.55 & 1.87 & 1.71 & 1.65 & 1.72 & 1.52 & 1.95 & 1.40 & 1.62 & 1.10 \\
2006 & 3.00 & 2.13 & 2.14 & 1.91 & 1.88 & 1.58 & 0.67 & 1.44 & 1.65 & 1.13 \\
2007 & 1.07 & 1.13 & 1.20 & 1.12 & 1.12 & 1.00 & 0.92 & 1.00 & 1.08 & 1.00 \\
2008 & 1.49 & 1.30 & 1.27 & 1.23 & 1.30 & 1.23 & 1.12 & 1.33 & 1.24 & 1.04 \\
2009 & 1.00 & 0.78 & 0.72 & 0.89 & 1.22 & 1.25 & 1.48 & 1.04 & 1.26 & 0.96 \\
\hline
\end{tabular}


Panel A (continued): Values of one-year Betas

(sorted: highest to lowest based on one-year mean beta values)

\begin{tabular}{ccccccccccc}
\hline & R18 & R11 & R01 & R05 & R14 & R17 & R20 & R09 & R08 & R03 \\
Year & RS1000 & SP100 & DJA & NYcomp & SPAero & SPHeal & GoldSil & NDInsr & NDbank & DJUtil \\
1990 & 1.02 & 0.96 & 0.96 & 0.97 & 1.02 & 1.03 & 0.13 & 1.25 & 0.81 & 0.39 \\
1991 & 0.99 & 0.96 & 0.90 & 0.95 & 0.77 & 1.21 & -0.55 & 0.71 & 0.72 & 0.37 \\
1992 & 1.00 & 0.92 & 0.77 & 0.94 & 0.95 & 1.42 & 0.10 & 0.61 & 0.69 & 1.08 \\
1993 & 1.01 & 0.90 & 0.84 & 0.98 & 0.16 & 0.64 & 0.49 & 0.63 & 0.69 & 0.67 \\
1994 & 0.98 & 1.01 & 1.19 & 0.97 & 0.70 & 1.03 & 0.05 & 0.80 & 0.53 & 0.87 \\
1995 & 0.98 & 1.14 & 1.49 & 1.03 & 1.92 & 0.70 & 2.87 & 0.38 & 0.10 & 0.71 \\
1996 & 0.99 & 1.03 & 0.86 & 0.89 & 0.50 & 1.30 & 0.51 & 0.77 & 0.50 & 0.79 \\
1997 & 0.97 & 1.09 & 1.09 & 0.90 & 1.11 & 1.05 & 0.66 & 0.47 & 0.59 & 0.35 \\
1998 & 1.03 & 0.99 & 0.96 & 0.99 & 1.16 & 0.81 & 1.54 & 1.02 & 1.08 & 0.25 \\
1999 & 1.00 & 1.12 & 0.88 & 0.82 & 0.53 & 0.57 & 0.26 & 0.46 & 0.48 & 0.14 \\
2000 & 1.01 & 1.05 & 0.84 & 0.79 & 0.55 & 0.01 & 0.04 & 0.61 & 0.41 & -0.09 \\
2001 & 1.02 & 1.08 & 0.94 & 0.76 & 0.95 & 0.22 & 0.08 & 0.02 & 0.31 & 0.10 \\
2002 & 0.98 & 1.08 & 1.02 & 0.85 & 0.27 & 0.62 & 0.19 & 0.41 & 0.35 & 0.44 \\
2003 & 0.98 & 0.97 & 0.97 & 1.08 & 1.71 & 0.39 & 0.44 & 0.76 & 0.73 & 0.97 \\
2004 & 1.05 & 0.93 & 0.90 & 1.06 & 0.61 & 0.91 & 0.38 & 1.03 & 0.91 & 0.52 \\
2005 & 1.03 & 0.85 & 1.08 & 0.93 & 0.70 & 0.45 & 1.72 & 0.86 & 0.93 & 0.02 \\
2006 & 1.03 & 0.88 & 0.74 & 1.13 & 0.64 & 0.42 & 3.07 & 0.49 & 0.48 & -0.07 \\
2007 & 1.00 & 1.03 & 1.02 & 1.01 & 0.80 & 0.91 & 1.05 & 0.85 & 0.69 & 0.59 \\
2008 & 1.04 & 0.89 & 0.82 & 1.09 & 1.24 & 0.76 & 0.98 & 0.82 & 0.54 & 0.62 \\
2009 & 1.00 & 0.97 & 0.98 & 1.10 & 1.17 & 0.68 & 0.74 & 0.97 & 1.25 & 0.51 \\
\hline
\end{tabular}

Table 4-B. Betas of Different Indices - Nonoverlapping Time Intervals

Panel B : Values of two-year Betas

(sorted: highest to lowest based on one-year mean beta values)

\begin{tabular}{lllllllllll}
\hline & R10 & R07 & R16 & R06 & R19 & R15 & R02 & R12 & R13 & R04 \\
Year & NDTel & ND100 & SpInfo & NDcomp & RS2000 & SPMatl & DJTrans & SP400 & SP600 & DJTotl \\
$1990-91$ & 1.33 & 1.53 & 1.00 & 1.29 & 1.18 & 1.06 & 1.39 & 1.18 & 1.16 & 1.01 \\
$1992-93$ & 1.61 & 1.28 & 1.34 & 1.04 & 0.86 & 0.67 & 0.83 & 1.04 & 0.94 & 1.00 \\
$1994-95$ & 1.25 & 1.00 & 0.90 & 0.98 & 0.90 & 1.16 & 1.37 & 0.98 & 1.00 & 0.99 \\
$1996-97$ & 0.90 & 1.42 & 1.59 & 1.08 & 0.67 & 0.86 & 0.84 & 0.82 & 0.71 & 0.98 \\
$1998-99$ & 1.61 & 1.49 & 1.36 & 1.46 & 1.14 & 0.88 & 0.92 & 1.18 & 1.09 & 1.02 \\
$2000-01$ & 1.82 & 2.12 & 2.21 & 1.82 & 0.79 & 0.87 & 1.06 & 1.01 & 0.81 & 1.02 \\
$2002-03$ & 1.73 & 1.52 & 1.73 & 1.32 & 1.00 & 1.06 & 0.86 & 0.87 & 0.85 & 0.98 \\
$2004-05$ & 1.75 & 1.78 & 1.72 & 1.71 & 1.75 & 1.35 & 1.54 & 1.36 & 1.58 & 1.04 \\
$2006-07$ & 1.58 & 1.31 & 1.37 & 1.28 & 1.33 & 1.10 & 0.85 & 1.09 & 1.24 & 1.01 \\
$2008-09$ & 1.25 & 1.09 & 1.07 & 1.09 & 1.20 & 1.28 & 1.18 & 1.16 & 1.18 & 1.02 \\
\hline
\end{tabular}

Panel B (Continued) : Values of two-year Betas

(sorted: highest to lowest based on one-year mean beta values)

\begin{tabular}{lllllllllll}
\hline & R18 & R11 & R01 & R05 & R14 & R17 & R20 & R09 & R08 & R03 \\
Year & RS1000 & SP100 & DJIA & NYcomp & SPAero & SPHeal & GoldSil & NDInsr & NDbank & DJUtil \\
$1990-91$ & 1.02 & 0.96 & 0.93 & 0.96 & 0.90 & 1.09 & -0.15 & 1.05 & 0.89 & 0.40 \\
$1992-93$ & 0.98 & 0.92 & 0.80 & 0.95 & 0.67 & 1.13 & 0.35 & 0.60 & 0.67 & 0.92 \\
$1994-95$ & 0.97 & 1.03 & 1.16 & 0.98 & 1.07 & 1.00 & 0.66 & 0.80 & 0.62 & 0.98 \\
$1996-97$ & 0.93 & 1.06 & 1.00 & 0.90 & 0.89 & 1.14 & 0.57 & 0.57 & 0.59 & 0.50 \\
$1998-99$ & 1.05 & 1.02 & 0.93 & 0.94 & 0.98 & 0.76 & 1.18 & 0.87 & 0.91 & 0.23 \\
$2000-01$ & 1.00 & 1.07 & 0.90 & 0.78 & 0.79 & 0.14 & 0.06 & 0.27 & 0.36 & 0.03 \\
$2002-03$ & 0.97 & 1.04 & 0.97 & 0.91 & 0.59 & 0.59 & 0.19 & 0.49 & 0.44 & 0.64 \\
$2004-05$ & 1.11 & 0.89 & 0.99 & 0.99 & 0.65 & 0.65 & 1.01 & 0.95 & 0.94 & 0.25 \\
$2006-07$ & 1.03 & 1.00 & 0.94 & 1.04 & 0.75 & 0.76 & 1.50 & 0.77 & 0.73 & 0.40 \\
$2008-09$ & 1.01 & 0.93 & 0.89 & 1.09 & 1.16 & 0.70 & 0.84 & 0.77 & 0.77 & 0.57 \\
\hline
\end{tabular}


Panel C : Values of four-year Betas

(sorted: highest to lowest based on one-year mean beta values)

\begin{tabular}{|c|c|c|c|c|c|c|c|c|c|c|}
\hline & R10 & R07 & R16 & R06 & R19 & R15 & R02 & R12 & R13 & R04 \\
\hline Year & NDTel & ND100 & SpInfo & NDcomp & RS2000 & SPMatl & DJTrans & SP400 & SP600 & DJTotl \\
\hline 1990-93 & 1.35 & 1.50 & 1.04 & 1.25 & 1.13 & 1.00 & 1.31 & 1.16 & 1.12 & 1.01 \\
\hline 1994-97 & 1.02 & 1.28 & 1.35 & 1.04 & 0.74 & 0.94 & 1.03 & 0.88 & 0.81 & 0.94 \\
\hline 1998-01 & 1.87 & 1.91 & 1.87 & 1.70 & 0.91 & 0.85 & 0.92 & 1.03 & 0.87 & 1.02 \\
\hline 2002-05 & 1.73 & 1.56 & 1.72 & 1.38 & 1.11 & 1.10 & 0.96 & 0.94 & 0.96 & 0.99 \\
\hline 2006-09 & 1.28 & 1.10 & 1.09 & 1.10 & 1.20 & 1.26 & 1.12 & 1.14 & 1.17 & 1.01 \\
\hline \multicolumn{11}{|c|}{$\begin{array}{l}\text { Panel C (Continued) : Values of four-year Betas } \\
\text { d: highest to lowest based on one-year mean beta values) }\end{array}$} \\
\hline & R18 & R11 & R01 & R05 & R14 & R17 & R20 & R09 & R08 & R03 \\
\hline Year & RS1000 & SP100 & DJIA & NYcomp & SPAero & SPHeal & GoldSil & NDInsr & NDbank & DJUtil \\
\hline 1990-93 & 1.01 & 0.95 & 0.91 & 0.96 & 0.86 & 1.12 & -0.11 & 0.98 & 0.84 & 0.47 \\
\hline 1994-97 & 0.98 & 1.05 & 1.05 & 0.93 & 0.92 & 1.08 & 0.56 & 0.63 & 0.62 & 0.66 \\
\hline 1998-01 & 1.02 & 1.06 & 0.90 & 0.83 & 0.81 & 0.42 & 0.59 & 0.50 & 0.53 & 0.11 \\
\hline 2002-05 & 0.99 & 1.01 & 0.97 & 0.93 & 0.60 & 0.60 & 0.29 & 0.55 & 0.50 & 0.60 \\
\hline 2006-09 & 1.02 & 0.94 & 0.90 & 1.09 & 1.12 & 0.70 & 0.89 & 0.76 & 0.76 & 0.58 \\
\hline
\end{tabular}

Table 4-C. Betas of Different Indices - Nonoverlapping Time Intervals

\section{Panel D : Values of five-year Betas}

(sorted: highest to lowest based on one-year mean beta values)

\begin{tabular}{lllllllllll}
\hline & R10 & R07 & R16 & R06 & R19 & R15 & R02 & R12 & R13 & R04 \\
Year & NDTel & ND100 & SpInfo & NDcomp & RS2000 & SPMatl & DJTrans & SP400 & SP600 & DJTotl \\
$1990-94$ & 1.33 & 1.45 & 1.05 & 1.22 & 1.10 & 1.04 & 1.32 & 1.14 & 1.10 & 1.01 \\
$1995-99$ & 1.33 & 1.42 & 1.40 & 1.30 & 0.97 & 0.90 & 0.91 & 1.05 & 0.96 & 1.01 \\
$2000-04$ & 1.82 & 1.83 & 1.96 & 1.59 & 0.92 & 0.97 & 0.97 & 0.94 & 0.85 & 0.99 \\
$2005-09$ & 1.29 & 1.14 & 1.12 & 1.12 & 1.22 & 1.27 & 1.16 & 1.15 & 1.19 & 1.02 \\
\hline
\end{tabular}

Panel D (Continued): Values of five-year Betas

(sorted: highest to lowest based on one-year mean beta values)

\begin{tabular}{lllllllllll}
\hline & R18 & R11 & R01 & R05 & R14 & R17 & R20 & R09 & R08 & R03 \\
Year & RS1000 & SP100 & DJIA & NYcomp & SPAero & SPHeal & GoldSil & NDInsr & NDbank & DJUtil \\
$1990-94$ & 1.01 & 0.96 & 0.94 & 0.96 & 0.85 & 1.10 & -0.07 & 0.96 & 0.80 & 0.53 \\
$1995-99$ & 1.01 & 1.04 & 0.97 & 0.93 & 0.99 & 0.91 & 1.01 & 0.75 & 0.79 & 0.35 \\
$2000-04$ & 1.00 & 1.05 & 0.93 & 0.85 & 0.69 & 0.36 & 0.15 & 0.40 & 0.41 & 0.34 \\
$2005-09$ & 1.02 & 0.94 & 0.91 & 1.08 & 1.11 & 0.69 & 0.93 & 0.77 & 0.77 & 0.56 \\
\hline
\end{tabular}

Panel E : Values of ten-year Betas

(sorted: highest to lowest based on one-year mean beta values)

\begin{tabular}{lllllllllll}
\hline & R18 & R11 & R01 & R05 & R14 & R17 & R20 & R09 & R08 & R03 \\
Year & RS1000 & SP100 & DJIA & NYcomp & SPAero & SPHeal & GoldSil & NDInsr & NDbank & DJUtil \\
$1990-99$ & 1.01 & 1.01 & 0.96 & 0.94 & 0.90 & 0.99 & 0.50 & 0.82 & 0.78 & 0.44 \\
$2000-09$ & 1.01 & 0.99 & 0.92 & 0.96 & 0.89 & 0.52 & 0.54 & 0.58 & 0.58 & 0.45 \\
\hline
\end{tabular}

Panel E (Continued) : Values of ten-year Betas

(sorted: highest to lowest based on one-year mean beta values)

\begin{tabular}{lllllllllll}
\hline & R10 & R07 & R16 & R06 & R19 & R15 & R02 & R12 & R13 & R04 \\
Year & NDTel & ND100 & SpInfo & NDcomp & RS2000 & SPMatl & DJTrans & SP400 & SP600 & DJTotl \\
$1990-99$ & 1.35 & 1.45 & 1.27 & 1.27 & 1.00 & 0.93 & 1.07 & 1.07 & 1.00 & 1.00 \\
$2000-09$ & 1.56 & 1.49 & 1.55 & 1.36 & 1.07 & 1.12 & 1.06 & 1.04 & 1.01 & 1.01 \\
\hline
\end{tabular}


Panel F : Values of Betas for the whole 20-year period

(sorted: highest to lowest based on one-year mean beta values)

\begin{tabular}{lllllllllll}
\hline & R10 & R07 & R16 & R06 & R19 & R15 & R02 & R12 & R13 & R04 \\
Year & NDTel & ND100 & SpInfo & NDcomp & RS2000 & SPMatl & DJTrans & SP400 & SP600 & DJTotl \\
$1990-09$ & 1.49 & 1.49 & 1.45 & 1.33 & 1.03 & 1.02 & 1.05 & 1.04 & 0.99 & 1.00 \\
\hline \multicolumn{8}{c}{$\begin{array}{c}\text { Panel F (Continued) : Values of the Betas for the whole 20-year period } \\
\text { (sorted: highest to lowest based on one-year mean beta values) }\end{array}$} \\
\hline Rear & R18 & R11 & R01 & R05 & R14 & R17 & R20 & R09 & R08 & R03 \\
1990-09 & 1.01 & SP100 & DJIA & NYcomp & SPAero & SPHeal & GoldSil & NDInsr & NDbank & DJUtil \\
\hline
\end{tabular}

Table 4-A shows the one-year beta values for the 20 indices for each of the 20 years, 1990 through 2009. The indices are sorted on the basis of the mean of the 20 one-year values. The beta values based on the two-year and four-year non-overlapping intervals are given in Table 4-B. Table 4-C contains the beta values based on the five-year and ten-year non-overlapping intervals as well as the whole 20-year period.

\section{Unit Root Tests for Betas}

A fundamental assumption underlying the Capital Asset Pricing Model is that the betas of the individual stocks or portfolios or indices remain constant. In Fama and Macbeth (1973), the beta values are calculated for periods of four years or longer so as to eliminate the estimation errors. A relevant question at this stage is whether the beta values are affected by the length of the estimation period. The means of the betas calculated from the individual values estimated using intervals of different lengths are given in Table 5.

Table 5. Means of Betas of Different Indices For Time Intervals of Different Lengths

\begin{tabular}{lcccccc}
\hline Index & $\begin{array}{c}\text { one-year } \\
\text { interval }\end{array}$ & $\begin{array}{c}\text { two-year } \\
\text { interval }\end{array}$ & $\begin{array}{c}\text { four-year } \\
\text { interval }\end{array}$ & $\begin{array}{c}\text { five-year } \\
\text { interval }\end{array}$ & $\begin{array}{c}\text { ten-year } \\
\text { interval }\end{array}$ & $\begin{array}{c}\text { 20-year } \\
\text { period }\end{array}$ \\
\hline R10_NDTel & 1.491 & 1.483 & 1.449 & 1.442 & 1.458 & 1.491 \\
R07_ND100 & 1.445 & 1.456 & 1.471 & 1.459 & 1.471 & 1.489 \\
R16_SPInfo & 1.430 & 1.429 & 1.415 & 1.382 & 1.409 & 1.446 \\
R06_NDComp & 1.309 & 1.308 & 1.294 & 1.308 & 1.314 & 1.330 \\
R19_RS2000 & 1.107 & 1.081 & 1.018 & 1.053 & 1.034 & 1.026 \\
R15_SPMatl & 1.103 & 1.028 & 1.029 & 1.044 & 1.025 & 1.021 \\
R02_DJTrans & 1.083 & 1.083 & 1.070 & 1.091 & 1.067 & 1.047 \\
R12_SP400 & 1.080 & 1.069 & 1.031 & 1.071 & 1.057 & 1.041 \\
R13_SP600 & 1.077 & 1.054 & 0.985 & 1.024 & 1.006 & 0.989 \\
R04_DJTotal & 1.009 & 1.007 & 0.997 & 1.007 & 1.005 & 1.003 \\
R18_RS1000 & 1.006 & 1.008 & 1.004 & 1.009 & 1.008 & 1.007 \\
R11_SP100 & 0.992 & 0.991 & 1.004 & 0.995 & 1.001 & 1.005 \\
R01_DJIA & 0.962 & 0.952 & 0.947 & 0.938 & 0.937 & 0.933 \\
R05_NYComp & 0.961 & 0.955 & 0.947 & 0.957 & 0.951 & 0.946 \\
R14_SPAero & 0.873 & 0.844 & 0.865 & 0.906 & 0.896 & 0.883 \\
R17_SPHealth & 0.757 & 0.796 & 0.784 & 0.765 & 0.757 & 0.720 \\
R20_GoldSil & 0.738 & 0.622 & 0.447 & 0.505 & 0.518 & 0.484 \\
R09_NDInsr & 0.696 & 0.712 & 0.686 & 0.720 & 0.699 & 0.668 \\
R08_NDBank & 0.639 & 0.690 & 0.650 & 0.691 & 0.678 & 0.666 \\
R03_DJUtil & 0.461 & 0.493 & 0.484 & 0.445 & 0.445 & 0.430 \\
\hline
\end{tabular}

One can see from Table 5 that the mean of each index is relatively constant (across the row), though the lengths of estimation intervals are significantly different from each other. Does this imply that the individual beta values are time-invariant or at least stationary? According to Jagannathan and Wang (1996), a primary weakness of the Capital Asset Pricing Model (CAPM) given by equation (1) is that it assumes that $\beta_{i}$ remains constant over time. The 
graphs in Figures 1 through 5 do show that the betas of the indices vary widely from one year to the next. In this context, the following two hypotheses are tested.

Hypothesis H1: The time-series of the one-year betas of each index is stationary.

Hypothesis H2: The time-series of the one-year betas of the indices grouped in each quintile are co-integrated.

The unit root tests are done only for the set of the one-year betas given in Table 4-A, that are estimated using one-year intervals. Since there are fewer values of betas in other cases (given in Tables 4-B, and 4-C), the unit root tests will not be reliable in those cases. The results of the unit root tests for the one-year betas are given in Table 6. In all cases, the augmented Dickey-Fuller unit root test for the level series (no first or second differences) with no intercept or trend option is used. All indices except the Gold and Silver index (series R20) have a unit root. In other words, all indices except the Gold and Silver index are non-stationary. This result makes hypothesis H1 invalid.

Table 6. Unit Root Tests for the One-year Betas of Different Indices

\begin{tabular}{lccc}
\hline Index & t-stat & p-value & unit root? \\
\hline R10_NDTel & -0.581 & 0.45 & Yes \\
R07_ND100 & -1.102 & 0.24 & Yes \\
R16_SPInfo & -1.051 & 0.25 & Yes \\
R06_NDComp & -0.909 & 0.31 & Yes \\
R19_RS2000 & -0.699 & 0.40 & Yes \\
R15_SPMatl & -0.741 & 0.36 & Yes \\
R02_DJTrans & 0.109 & 0.70 & Yes \\
R12_SP400 & -0.242 & 0.59 & Yes \\
R13_SP600 & -0.652 & 0.42 & Yes \\
R04_DJTotal & -0.138 & 0.62 & Yes \\
R18_RS1000 & 0.124 & 0.71 & Yes \\
R11_SP100 & -0.152 & 0.62 & Yes \\
R01_DJIA & -0.291 & 0.57 & Yes \\
R05_NYComp & 0.073 & 0.69 & Yes \\
R14_SPAero & -0.228 & 0.59 & Yes \\
R17_SPHealth & -1.221 & 0.19 & Yes \\
R20_GoldSil & -2.286 & 0.025 & No \\
R09_NDInsr & -0.276 & 0.57 & Yes \\
R08_NDBank & 0.324 & 0.76 & Yes \\
R03_DJUtil & -1.307 & 0.17 & Yes \\
\hline
\end{tabular}

The results related to the Johansen co-integration tests are given in Table 7. The results in Table 7 are interesting. The indices in the middle quintile which have betas close to 1 are not co-integrated, whereas the indices in other quintiles are co-integrated. Perhaps, the returns of S\&P 600 Small Cap Index follow a pattern different from that of the three other indices in the middle quintile. These results prove that hypothesis $\mathrm{H} 2$ is valid in four of the five cases.

Table 7. Co-integration Tests for One-year Betas Grouped into Quintiles

\begin{tabular}{clccc}
\hline Quintile & Indices in the Quintile & $\begin{array}{c}\text { Max-Eigen } \\
\text { Statistic }\end{array}$ & p- value & $\begin{array}{c}\text { At least One } \\
\text { Co-integrating Equation? }\end{array}$ \\
\hline 1 & $\begin{array}{l}\text { R10_NDTEL, R07_ND100 } \\
\text { R16_SPINFO, R06_NDCOMP }\end{array}$ & 26.75 & 0.022 & Yes \\
2 & $\begin{array}{l}\text { R19_RS2000, R15_SPMATL } \\
\text { R02_DJTRANS, R12_SP400 }\end{array}$ & 24.05 & 0.052 & Yes \\
3 & $\begin{array}{l}\text { R13_SP600, R04_DJTOTAL } \\
\text { R18_RS1000, R11_SP100 }\end{array}$ & 8.59 & 0.97 & No \\
4 & $\begin{array}{l}\text { R01_DJIA, R05_NYCOMP } \\
\text { R14_SPAERO, R17_SPHEALTH }\end{array}$ & 25.11 & 0.037 & Yes \\
& $\begin{array}{l}\text { R20_GOLDSIL, R09_NDINSR } \\
\text { R08_NDBANK,R03_DJUTIL }\end{array}$ & 28.30 & 0.013 & Yes \\
\hline
\end{tabular}


Table 8-A. Values of One-Year Betas and Standard Deviations of the Error Terms

\begin{tabular}{|c|c|c|c|c|c|c|c|c|c|c|}
\hline \multicolumn{11}{|c|}{ Estimates for the one-year intervals covering the years 1990 through 1994} \\
\hline & \multicolumn{2}{|c|}{1990} & \multicolumn{2}{|c|}{1991} & \multicolumn{2}{|c|}{1992} & \multicolumn{2}{|c|}{1993} & \multicolumn{2}{|c|}{1994} \\
\hline Index & Beta & $\operatorname{Std}(\varepsilon)$ & Beta & $\operatorname{Std}(\varepsilon)$ & Beta & $\operatorname{Std}(\varepsilon)$ & Beta & $\operatorname{Std}(\varepsilon)$ & Beta & $\operatorname{Std}(\varepsilon)$ \\
\hline R01 & 0.962 & 0.0092 & 0.905 & 0.0097 & 0.767 & 0.0158 & 0.838 & 0.0111 & 1.193 & 0.0089 \\
\hline R02 & 1.245 & 0.0421 & 1.445 & 0.0483 & 1.070 & 0.0361 & 0.416 & 0.0260 & 1.290 & 0.0231 \\
\hline R03 & 0.394 & 0.0354 & 0.375 & 0.0274 & 1.076 & 0.0244 & 0.666 & 0.0332 & 0.867 & 0.0307 \\
\hline R04 & 1.032 & 0.0044 & 0.976 & 0.0034 & 0.940 & 0.0085 & 1.042 & 0.0059 & 0.952 & 0.0037 \\
\hline R05 & 0.966 & 0.0022 & 0.945 & 0.0021 & 0.941 & 0.0027 & 0.975 & 0.0033 & 0.967 & 0.0029 \\
\hline R06 & .305 & 0.0221 & 1.125 & 0.0206 & 0.799 & 0.0401 & 1.439 & 0.0242 & 0.953 & 0.0169 \\
\hline R07 & .559 & 0.0227 & 1.414 & 0.0341 & 1.036 & 0.0402 & 1.681 & 0.0324 & 1.126 & 0.0244 \\
\hline R08 & 0.815 & 0.0317 & 0.721 & 0.0311 & 0.689 & 0.0237 & 0.695 & 0.0414 & 0.526 & 0.0316 \\
\hline R09 & .248 & 0.0349 & 0.710 & 0.0245 & 0.614 & 0.0291 & 0.634 & 0.0310 & 0.798 & 0.0185 \\
\hline R10 & .475 & 0.0262 & 0.996 & 0.0323 & 1.004 & 0.0374 & 2.498 & 0.0366 & 1.103 & 0.0266 \\
\hline R11 & 0.963 & 0.0046 & 0.955 & 0.0066 & 0.923 & 0.0050 & 0.902 & 0.0044 & 1.012 & 0.0056 \\
\hline R12 & 1.219 & 0.0181 & 1.069 & 0.0118 & 0.991 & & 1.123 & 0.0178 & 0.951 & 0.0112 \\
\hline R13 & .194 & 0.0255 & 0.943 & 0.0220 & 0.696 & 0.0421 & 1.333 & 0.0245 & 0.940 & 0.0232 \\
\hline R14 & .021 & 0.0241 & 0.773 & & 0.954 & & 0.160 & & 0.701 & 0.0230 \\
\hline R15 & .124 & 0.0213 & 0.954 & 0.0297 & 0.826 & 0.0227 & 0.421 & 0.0238 & 1.283 & 0.0294 \\
\hline R16 & 128 & 0.0396 & 0.970 & & & & 1.874 & & 1.228 & 0.0362 \\
\hline R17 & .031 & 0.0247 & 1.211 & 0.0165 & 1.419 & 0.02 & 0.644 & 0.0 & 1.028 & 0.0376 \\
\hline R18 & 020 & 0.0030 & 0.990 & & & & 1.007 & & 0.976 & 0.0018 \\
\hline R19 & .226 & 0.0252 & 0.985 & 0.0276 & 0.699 & 0.04 & 1.118 & 0.0211 & 0.872 & 0.0178 \\
\hline R20 & 128 & 0.0971 & -0.554 & 0.0795 & 0.105 & 0.0742 & 0.493 & 0.1012 & 0.045 & 0.0873 \\
\hline \multicolumn{11}{|c|}{ Estimates for the one-year intervals covering the years 1995 through1999 } \\
\hline & \multicolumn{2}{|c|}{1995} & \multicolumn{2}{|c|}{1996} & \multicolumn{2}{|c|}{1997} & \multicolumn{2}{|c|}{1998} & \multicolumn{2}{|c|}{1999} \\
\hline Index & Beta & $\operatorname{Std}(\varepsilon$ & Beta & $\operatorname{Std}(\varepsilon$ & Beta & & Beta & & Beta & $\operatorname{Std}(\varepsilon)$ \\
\hline R01 & 1.489 & 0.0117 & 0.863 & 0.0 & 1.089 & 0.0 & 0.960 & & 0.876 & 0.0260 \\
\hline R02 & 1.310 & 0.0337 & 0.913 & & & & 0.931 & & 0.893 & 0.0429 \\
\hline R03 & 0.712 & 0.0330 & 0.791 & 0.0322 & 0.348 & 0.0291 & 0.248 & 0.0402 & 0.141 & 0.0453 \\
\hline R04 & 0.961 & 0.0058 & 0.952 & & & & 1.059 & & 1.055 & 0.0075 \\
\hline R05 & 1.035 & 0.0033 & 0.893 & 0.0038 & 0.899 & 0.0060 & 0.986 & 0.0060 & 0.816 & 0.0125 \\
\hline R06 & 0.723 & 0.0266 & 1.147 & 0.0374 & 1.066 & 0.0373 & 1.370 & 0.0326 & 1.780 & 0.0525 \\
\hline R07 & 0.301 & 0.0373 & 1.457 & 0.0325 & 1.445 & 0.0507 & 1.346 & 0.0554 & 1.919 & 0.0603 \\
\hline R08 & 0.095 & 0.0240 & 0.505 & 0.0119 & 0.595 & 0.0346 & 1.078 & 0.0285 & 0.477 & 0.0359 \\
\hline R09 & 0.375 & 0.0193 & 0.773 & 0.0293 & 0.466 & 0.0332 & 1.023 & 0.0195 & 0.464 & 0.0620 \\
\hline R10 & 1.395 & & & & & & 1.565 & 0.0399 & 1.808 & 0.0606 \\
\hline R11 & 1.143 & 0.0055 & 1.026 & 0.0032 & 1.085 & 0.0071 & 0.992 & 0.0090 & 1.117 & 0.0067 \\
\hline R12 & 1.063 & 0.0171 & 0.861 & 0.0222 & 0.799 & 0.0265 & 1.294 & 0.0258 & 0.889 & 0.0335 \\
\hline R13 & 1.216 & 0.0276 & 0.816 & 0.0321 & 0.659 & 0.0393 & 1.234 & 0.0213 & 0.738 & 0.0414 \\
\hline R14 & 1.923 & 0.0170 & 0.504 & 0.0163 & 1.111 & 0.0351 & 1.157 & 0.0580 & 0.531 & 0.0674 \\
\hline R15 & 1.860 & 0.0298 & 0.769 & 0.0306 & 0.919 & 0.0245 & 0.800 & 0.0384 & 1.173 & 0.0748 \\
\hline R16 & 0.153 & 0.0524 & 1.704 & 0.0411 & 1.572 & 0.0559 & 1.249 & 0.0456 & 1.678 & 0.0676 \\
\hline R17 & 0.700 & 0.0228 & 1.305 & 0.0196 & 1.053 & 0.0352 & 0.806 & 0.0300 & 0.573 & 0.0596 \\
\hline R18 & 0.980 & 0.0032 & 0.993 & 0.0034 & 0.973 & 0.0040 & 1.030 & 0.0027 & 1.004 & 0.0035 \\
\hline R19 & 1.073 & 0.0241 & 0.793 & 0.0368 & 0.614 & 0.0390 & 1.230 & 0.0222 & 0.945 & 0.0422 \\
\hline R20 & 2.869 & 0.0806 & 0.510 & 0.0807 & 0.663 & 0.1169 & 1.545 & 0.1722 & 0.255 & 0.1252 \\
\hline
\end{tabular}


Table 8-A (Continued). Values of One-Year Betas and Standard Deviations of the Error Terms

\begin{tabular}{|c|c|c|c|c|c|c|c|c|c|c|}
\hline \multicolumn{11}{|c|}{ Estimates for the one-year intervals covering the years 2000 through 2004} \\
\hline & \multicolumn{2}{|c|}{2000} & \multicolumn{2}{|c|}{2001} & \multicolumn{2}{|c|}{2002} & \multicolumn{2}{|c|}{2003} & \multicolumn{2}{|c|}{2004} \\
\hline Index & Beta & $\operatorname{Std}(\varepsilon)$ & Beta & $\operatorname{Std}(\varepsilon)$ & Beta & $\operatorname{Std}(\varepsilon)$ & Beta & $\operatorname{Std}(\varepsilon)$ & Beta & $\operatorname{Std}(\varepsilon)$ \\
\hline R01 & 0.839 & 0.0269 & 0.944 & 0.0226 & 1.015 & 0.0167 & 0.975 & 0.0104 & 0.902 & 0.0099 \\
\hline R02 & 0.846 & 0.0774 & 1.211 & 0.0575 & 0.691 & 0.0425 & 1.459 & 0.0349 & 1.036 & 0.0347 \\
\hline R03 & -0.093 & 0.0705 & 0.101 & 0.0551 & 0.438 & 0.0656 & 0.970 & 0.0405 & 0.517 & 0.0191 \\
\hline R04 & 0.942 & 0.0229 & 1.049 & 0.0052 & 0.947 & 0.0054 & 1.004 & 0.0045 & 1.122 & 0.0031 \\
\hline R05 & 0.787 & 0.0200 & 0.763 & 0.0107 & 0.852 & 0.0093 & 1.077 & 0.0075 & 1.065 & 0.0053 \\
\hline R06 & 1.366 & 0.1163 & 2.170 & 0.0435 & 1.375 & 0.0333 & 0.974 & 0.0229 & 1.791 & 0.0193 \\
\hline R07 & 1.582 & 0.1072 & 2.532 & 0.0549 & 1.663 & 0.0455 & 0.934 & 0.0247 & 1.683 & 0.0218 \\
\hline R08 & 0.412 & 0.0577 & 0.312 & 0.0281 & 0.346 & 0.0285 & 0.730 & 0.0211 & 0.907 & 0.0200 \\
\hline R09 & 0.606 & 0.0606 & 0.017 & 0.0350 & 0.414 & 0.0375 & 0.764 & 0.0137 & 1.028 & 0.0146 \\
\hline R10 & 1.272 & 0.1182 & 2.242 & 0.0610 & 1.792 & 0.0802 & 0.894 & 0.0334 & 1.968 & 0.0267 \\
\hline R11 & 1.054 & 0.0124 & 1.084 & 0.0070 & 1.076 & 0.0102 & 0.965 & 0.0069 & 0.927 & 0.0054 \\
\hline $\mathrm{R} 12$ & 0.915 & 0.0380 & 1.076 & 0.0264 & 0.811 & 0.0250 & 1.005 & 0.0203 & 1.321 & 0.0107 \\
\hline R13 & 0.559 & 0.0707 & 0.988 & 0.0328 & 0.729 & 0.0426 & 1.145 & 0.0242 & 1.514 & 0.0221 \\
\hline R14 & 0.552 & 0.0902 & 0.949 & 0.0686 & 0.266 & 0.0532 & 1.707 & 0.0356 & 0.607 & 0.0340 \\
\hline R15 & 0.914 & 0.0781 & 0.839 & 0.0454 & 1.027 & 0.0397 & 1.429 & 0.0281 & 1.154 & 0.0330 \\
\hline R16 & 1.772 & 0.0842 & 2.540 & 0.0639 & 1.994 & 0.0508 & 0.960 & 0.0244 & 1.766 & 0.0293 \\
\hline R17 & 0.013 & 0.0590 & 0.217 & 0.0450 & 0.623 & 0.0210 & 0.387 & 0.0263 & 0.909 & 0.0263 \\
\hline R18 & 1.011 & 0.0094 & 1.020 & 0.0023 & 0.978 & 0.0025 & 0.984 & 0.0024 & 1.048 & 0.0013 \\
\hline R19 & 0.441 & 0.0829 & 1.048 & 0.0357 & 0.876 & 0.0431 & 1.220 & 0.0254 & 1.760 & 0.0212 \\
\hline R20 & 0.045 & 0.0776 & 0.082 & 0.0760 & 0.191 & 0.1238 & 0.444 & 0.0719 & 0.383 & 0.0987 \\
\hline \multicolumn{11}{|c|}{ Estimates for the one-year intervals covering the years 2005 through 2009} \\
\hline & \multicolumn{2}{|c|}{2005} & \multicolumn{2}{|c|}{2006} & \multicolumn{2}{|c|}{2007} & \multicolumn{2}{|c|}{2008} & \multicolumn{2}{|c|}{2009} \\
\hline Index & Beta & $\operatorname{Std}(\varepsilon)$ & Beta & $\operatorname{Std}(\varepsilon)$ & Beta & $\operatorname{Std}(\varepsilon)$ & Beta & $\operatorname{Std}(\varepsilon)$ & Beta & $\operatorname{Std}(\varepsilon)$ \\
\hline R01 & 1.077 & 0.0070 & 0.739 & 0.0065 & 1.016 & 0.0089 & 0.823 & 0.0146 & 0.975 & 0.0120 \\
\hline R02 & 1.946 & 0.0274 & 0.666 & 0.0486 & 0.915 & 0.0309 & 1.116 & 0.0361 & 1.478 & 0.0292 \\
\hline R03 & 0.019 & 0.0374 & -0.065 & 0.0315 & 0.590 & 0.0324 & 0.616 & 0.0409 & 0.513 & 0.0363 \\
\hline R04 & 1.104 & 0.0029 & 1.129 & 0.0051 & 0.999 & 0.0036 & 1.039 & 0.0053 & 0.959 & 0.0071 \\
\hline R05 & 0.931 & 0.0100 & 1.129 & 0.0100 & 1.009 & 0.0066 & 1.093 & 0.0140 & 1.095 & 0.0134 \\
\hline R06 & 1.652 & 0.0164 & 1.905 & 0.0165 & 1.120 & 0.0164 & 1.235 & 0.0190 & 0.893 & 0.0236 \\
\hline R07 & 1.868 & 0.0217 & 2.132 & 0.0188 & 1.131 & 0.0200 & 1.300 & 0.0299 & 0.783 & 0.0245 \\
\hline R08 & 0.926 & 0.0233 & 0.475 & 0.0077 & 0.689 & 0.0261 & 0.542 & 0.0699 & 1.247 & 0.0364 \\
\hline R09 & 0.857 & 0.0175 & 0.489 & 0.0106 & 0.847 & 0.0221 & 0.816 & 0.0494 & 0.974 & 0.0367 \\
\hline R10 & 1.547 & 0.0274 & 2.999 & 0.0575 & 1.075 & 0.0348 & 1.494 & 0.0335 & 1.002 & 0.0484 \\
\hline R11 & 0.851 & 0.0045 & 0.881 & 0.0051 & 1.029 & 0.0054 & 0.888 & 0.0095 & 0.973 & 0.0071 \\
\hline R12 & 1.404 & 0.0098 & 1.436 & 0.0178 & 1.004 & 0.0146 & 1.331 & 0.0175 & 1.039 & 0.0258 \\
\hline R13 & 1.618 & 0.0156 & 1.647 & 0.0253 & 1.083 & 0.0160 & 1.243 & 0.0239 & 1.256 & 0.0386 \\
\hline R14 & 0.699 & 0.0187 & 0.641 & 0.0197 & 0.801 & 0.0245 & 1.236 & 0.0376 & 1.168 & 0.0451 \\
\hline R15 & 1.524 & 0.0322 & 1.575 & 0.0232 & 0.999 & 0.0226 & 1.229 & 0.0397 & 1.250 & 0.0347 \\
\hline R16 & 1.713 & 0.0180 & 2.138 & 0.0235 & 1.196 & 0.0234 & 1.271 & 0.0267 & 0.724 & 0.0268 \\
\hline R17 & 0.451 & 0.0209 & 0.424 & 0.0227 & 0.912 & 0.0230 & 0.757 & 0.0310 & 0.680 & 0.0384 \\
\hline R18 & 1.029 & 0.0012 & 1.032 & 0.0018 & 1.004 & 0.0020 & 1.042 & 0.0033 & 0.997 & 0.0028 \\
\hline R19 & 1.723 & 0.0164 & 1.875 & 0.0255 & 1.117 & 0.0184 & 1.301 & 0.0243 & 1.221 & 0.0326 \\
\hline R20 & 1.719 & 0.0816 & 3.073 & 0.0940 & 1.046 & 0.0778 & 0.978 & 0.1713 & 0.737 & 0.1344 \\
\hline
\end{tabular}


Table 8-B. Values of Three-Year Betas and Standard Deviations of the Error Terms

\begin{tabular}{|c|c|c|c|c|c|c|c|c|c|c|}
\hline \multicolumn{11}{|c|}{ Estimates for the three-year intervals covering the years 1990 through 1996} \\
\hline & \multicolumn{2}{|c|}{ 1990-1992 } & \multicolumn{2}{|c|}{ 1991-1993 } & \multicolumn{2}{|c|}{ 1992-1994 } & \multicolumn{2}{|c|}{ 1993-1995 } & \multicolumn{2}{|c|}{ 1994-1996 } \\
\hline Index & Beta & $\operatorname{Std}(\varepsilon)$ & Beta & $\operatorname{Std}(\varepsilon)$ & Beta & $\operatorname{Std}(\varepsilon)$ & Beta & $\operatorname{Std}(\varepsilon)$ & Beta & $\operatorname{Std}(\varepsilon)$ \\
\hline R01 & 0.912 & 0.0116 & 0.859 & 0.0122 & 1.016 & 0.0126 & 1.096 & 0.0114 & 1.045 & 0.0119 \\
\hline R02 & 1.361 & 0.0413 & 1.299 & 0.0372 & 1.133 & 0.0300 & 1.171 & 0.0291 & 1.192 & 0.0286 \\
\hline R03 & 0.456 & 0.0289 & 0.512 & 0.0280 & 0.927 & 0.0289 & 0.909 & 0.0314 & 0.907 & 0.0307 \\
\hline R04 & 1.013 & 0.0061 & 0.983 & 0.0060 & 0.970 & 0.0061 & 0.976 & 0.0051 & 0.962 & 0.0064 \\
\hline R05 & 0.962 & 0.0025 & 0.951 & 0.0027 & 0.967 & 0.0030 & 0.975 & 0.0032 & 0.948 & 0.0034 \\
\hline R06 & 1.244 & 0.0296 & 1.140 & 0.0286 & 1.015 & 0.0278 & 1.040 & 0.0223 & 1.046 & 0.0268 \\
\hline R07 & 1.494 & 0.0323 & 1.434 & 0.0346 & 1.196 & 0.0314 & 1.113 & 0.0312 & 1.193 & 0.0317 \\
\hline R08 & 0.846 & 0.0384 & 0.660 & 0.0320 & 0.649 & 0.0338 & 0.606 & 0.0323 & 0.574 & 0.0238 \\
\hline R09 & 1.002 & 0.0328 & 0.665 & 0.0274 & 0.743 & 0.0268 & 0.769 & 0.0228 & 0.783 & 0.0223 \\
\hline R10 & 1.292 & 0.0352 & 1.092 & 0.0377 & 1.398 & 0.0377 & 1.362 & 0.0394 & 1.060 & 0.0350 \\
\hline R11 & 0.954 & 0.0052 & 0.944 & 0.0052 & 0.967 & 0.0050 & 1.013 & 0.0051 & 1.031 & 0.0047 \\
\hline R12 & 1.165 & 0.0176 & 1.092 & 0.0171 & 1.009 & 0.0169 & 0.984 & 0.0152 & 0.934 & 0.0165 \\
\hline R13 & 1.113 & 0.0330 & 0.954 & 0.0295 & 0.973 & 0.0304 & 1.022 & 0.0246 & 0.931 & 0.0264 \\
\hline R14 & 0.903 & 0.0269 & 0.718 & 0.0274 & 0.703 & 0.0275 & 0.913 & 0.0240 & 0.848 & 0.0223 \\
\hline R15 & 1.033 & 0.0240 & 0.864 & 0.0246 & 1.004 & 0.0252 & 1.023 & 0.0290 & 1.003 & 0.0304 \\
\hline R16 & 0.998 & 0.0372 & 0.997 & & 1.243 & 0.0355 & 1.056 & 0.0416 & 1.221 & 0.0437 \\
\hline R17 & 1.140 & 0.0283 & 1.301 & 0.0347 & 1.006 & 0.0389 & 1.005 & 0.0378 & 1.105 & 0.0272 \\
\hline R18 & 1.012 & 0.0029 & 0.998 & 0.0032 & 0.992 & 0.0032 & 0.993 & 0.0031 & 0.992 & 0.0028 \\
\hline R19 & 1.133 & 0.0332 & 0.964 & 0.0300 & 0.892 & 0.0281 & 0.909 & 0.0207 & 0.857 & 0.0258 \\
\hline R20 & -0.131 & 0.0803 & -0.432 & 0.0871 & 0.266 & 0.0890 & 0.485 & 0.0922 & 0.600 & 0.0809 \\
\hline \multicolumn{11}{|c|}{ Estimates for the three-year intervals covering the years 1995 through 2001} \\
\hline & \multicolumn{2}{|c|}{$1995-1997$} & \multicolumn{2}{|c|}{ 1996-1998 } & \multicolumn{2}{|c|}{ 1997-1999 } & \multicolumn{2}{|c|}{$1998-2000$} & \multicolumn{2}{|c|}{ 1999-2001 } \\
\hline Index & Beta & $\operatorname{Std}(\varepsilon)$ & Beta & $\operatorname{Std}(\varepsilon)$ & Beta & $\operatorname{Std}(\varepsilon)$ & Beta & $\operatorname{Std}(\varepsilon)$ & Beta & $\operatorname{Std}(\varepsilon)$ \\
\hline $\mathrm{R} 01$ & 1.037 & 0.0124 & 0.979 & 0.0157 & 0.974 & 0.0201 & 0.896 & 0.0241 & 0.899 & 0.0239 \\
\hline R02 & 0.873 & 0.0309 & 0.886 & 0.0335 & 0.894 & 0.0378 & 0.830 & 0.0535 & 0.960 & 0.0593 \\
\hline R03 & 0.520 & 0.0307 & 0.360 & 0.0334 & 0.269 & 0.0377 & 0.047 & 0.0541 & 0.025 & 0.0604 \\
\hline R04 & 0.931 & 0.0079 & 1.000 & 0.0084 & 1.015 & 0.0080 & 1.020 & 0.0138 & 1.017 & 0.0137 \\
\hline R05 & 0.907 & 0.0044 & 0.946 & 0.0062 & 0.932 & 0.0095 & 0.859 & 0.0155 & 0.764 & 0.0150 \\
\hline R06 & 1.062 & 0.0326 & 1.242 & 0.0350 & 1.333 & 0.0459 & 1.522 & 0.0754 & 1.892 & 0.0775 \\
\hline R07 & 1.342 & 0.0407 & 1.383 & 0.0477 & 1.454 & 0.0588 & 1.637 & 0.0779 & 2.173 & 0.0782 \\
\hline R08 & 0.556 & 0.0253 & 0.852 & 0.0349 & 0.842 & 0.0405 & 0.659 & 0.0471 & 0.318 & 0.0419 \\
\hline R09 & 0.566 & 0.0272 & 0.816 & 0.0295 & 0.756 & 0.0423 & 0.712 & 0.0519 & 0.282 & 0.0528 \\
\hline R10 & 0.932 & 0.0400 & 1.270 & 0.0442 & 1.396 & 0.0522 & 1.672 & 0.0835 & 1.984 & 0.0884 \\
\hline R11 & 1.068 & 0.0057 & 1.023 & 0.0071 & 1.037 & 0.0088 & 1.050 & 0.0103 & 1.100 & 0.0095 \\
\hline R12 & 0.837 & 0.0211 & 1.082 & 0.0260 & 1.075 & 0.0294 & 1.031 & 0.0351 & 0.938 & 0.0330 \\
\hline R13 & 0.739 & 0.0317 & 0.994 & 0.0337 & 0.971 & 0.0364 & 0.853 & 0.0508 & 0.749 & 0.0495 \\
\hline R14 & 0.978 & 0.0299 & 1.032 & 0.0415 & 1.018 & 0.0533 & 0.745 & 0.0733 & 0.690 & 0.0741 \\
\hline R15 & 0.922 & 0.0285 & 0.822 & 0.0312 & 0.890 & 0.0498 & 0.884 & 0.0640 & 0.935 & 0.0643 \\
\hline R16 & 1.484 & 0.0495 & 1.404 & 0.0477 & 1.403 & 0.0572 & 1.607 & 0.0690 & 2.162 & 0.0728 \\
\hline R17 & 1.123 & 0.0261 & 0.958 & 0.0289 & 0.853 & 0.0445 & 0.462 & 0.0550 & 0.179 & 0.0555 \\
\hline R18 & 0.980 & 0.0034 & 1.007 & 0.0035 & 1.008 & 0.0035 & 1.013 & 0.0058 & 1.012 & 0.0057 \\
\hline R19 & 0.698 & 0.0322 & 0.975 & 0.0351 & 0.990 & 0.0371 & 0.882 & 0.0559 & 0.807 & 0.0557 \\
\hline R20 & 0.742 & 0.0925 & 1.109 & 0.1244 & 1.015 & 0.1360 & 0.837 & 0.1281 & 0.133 & 0.0910 \\
\hline
\end{tabular}


Table8-B. (Continued) Values of Three-Year Betas and Standard Deviations of the Error Terms

\begin{tabular}{|c|c|c|c|c|c|c|c|c|c|c|}
\hline \multicolumn{11}{|c|}{ Estimates for the three-year intervals covering the years 2000 through 2006} \\
\hline & \multicolumn{2}{|c|}{$2000-2002$} & \multicolumn{2}{|c|}{$2001-2003$} & \multicolumn{2}{|c|}{$2002-2004$} & \multicolumn{2}{|c|}{ 2003-2005 } & \multicolumn{2}{|c|}{$2004-2006$} \\
\hline Index & Beta & $\operatorname{Std}(\varepsilon)$ & Beta & $\operatorname{Std}(\varepsilon)$ & Beta & $\operatorname{Std}(\varepsilon)$ & Beta & $\operatorname{Std}(\varepsilon)$ & Beta & $\operatorname{Std}(\varepsilon)$ \\
\hline R01 & 0.942 & 0.0215 & 0.961 & 0.0166 & 0.962 & 0.0129 & 0.999 & 0.0090 & 0.956 & 0.0085 \\
\hline R02 & 0.915 & 0.0585 & 0.994 & 0.0459 & 0.881 & 0.0377 & 1.426 & 0.0323 & 1.295 & 0.0379 \\
\hline R03 & 0.209 & 0.0674 & 0.456 & 0.0554 & 0.648 & 0.0454 & 0.592 & 0.0334 & 0.155 & 0.0292 \\
\hline R04 & 0.981 & 0.0134 & 1.000 & 0.0054 & 0.981 & 0.0047 & 1.054 & 0.0036 & 1.109 & 0.0037 \\
\hline R05 & 0.809 & 0.0141 & 0.859 & 0.0103 & 0.926 & 0.0082 & 1.028 & 0.0076 & 1.022 & 0.0084 \\
\hline R06 & 1.639 & 0.0738 & 1.639 & 0.0397 & 1.353 & 0.0262 & 1.371 & 0.0214 & 1.731 & 0.0168 \\
\hline R07 & 1.935 & 0.0743 & 1.901 & 0.0501 & 1.531 & 0.0329 & 1.375 & 0.0244 & 1.822 & 0.0204 \\
\hline R08 & 0.354 & 0.0382 & 0.392 & 0.0256 & 0.466 & 0.0239 & 0.864 & 0.0209 & 0.841 & 0.0179 \\
\hline R09 & 0.331 & 0.0453 & 0.309 & 0.0318 & 0.528 & 0.0246 & 0.811 & 0.0152 & 0.822 & 0.0147 \\
\hline R10 & 1.798 & 0.0873 & 1.936 & 0.0633 & 1.743 & 0.0533 & 1.421 & 0.0321 & 2.062 & 0.0394 \\
\hline R11 & 1.070 & 0.0096 & 1.054 & 0.0081 & 1.026 & 0.0079 & 0.942 & 0.0056 & 0.904 & 0.0055 \\
\hline R12 & 0.942 & 0.0299 & 0.944 & 0.0235 & 0.902 & 0.0194 & 1.154 & 0.0144 & 1.336 & 0.0137 \\
\hline R13 & 0.787 & 0.0494 & 0.896 & 0.0332 & 0.903 & 0.0310 & 1.328 & 0.0206 & 1.564 & 0.0206 \\
\hline R14 & 0.589 & 0.0712 & 0.738 & 0.0572 & 0.596 & 0.0459 & 1.150 & 0.0321 & 0.656 & 0.0237 \\
\hline R15 & 0.918 & 0.0545 & 0.978 & 0.0375 & 1.060 & 0.0334 & 1.390 & 0.0295 & 1.398 & 0.0282 \\
\hline R16 & 2.109 & 0.0675 & 2.024 & 0.0537 & 1.718 & 0.0388 & 1.373 & 0.0255 & 1.794 & 0.0232 \\
\hline R17 & 0.342 & 0.0478 & 0.453 & 0.0323 & 0.613 & 0.0238 & 0.512 & 0.0239 & 0.593 & 0.0226 \\
\hline R18 & 1.002 & 0.0056 & 0.999 & 0.0025 & 0.989 & 0.0022 & 1.008 & 0.0018 & 1.031 & 0.0015 \\
\hline R19 & 0.830 & 0.0561 & 1.015 & 0.0343 & 1.058 & 0.0316 & 1.476 & 0.0211 & 1.758 & 0.0203 \\
\hline R20 & 0.072 & 0.0925 & 0.173 & 0.0890 & 0.174 & 0.0963 & 0.762 & 0.0821 & 1.447 & 0.0897 \\
\hline \multicolumn{11}{|c|}{ Estimates for the three-year intervals covering the years 2005 through 2008} \\
\hline & \multicolumn{2}{|c|}{$2005-2007$} & \multicolumn{2}{|c|}{$2006-2008$} & & & & & & \\
\hline Index & Beta & $\operatorname{Std}(\varepsilon)$ & Beta & $\operatorname{Std}(\varepsilon)$ & & & & & & \\
\hline R01 & 0.997 & 0.0079 & 0.869 & 0.0105 & & & & & & \\
\hline R02 & 1.183 & 0.0369 & 0.927 & 0.0386 & & & & & & \\
\hline R03 & 0.266 & 0.0327 & 0.644 & 0.0343 & & & & & & \\
\hline R04 & 1.052 & 0.0040 & 1.038 & 0.0046 & & & & & & \\
\hline R05 & 1.006 & 0.0086 & 1.091 & 0.0101 & & & & & & \\
\hline R06 & 1.401 & 0.0177 & 1.203 & 0.0184 & & & & & & \\
\hline R07 & 1.495 & 0.0229 & 1.266 & 0.0249 & & & & & & \\
\hline R08 & 0.779 & 0.0222 & 0.521 & 0.0424 & & & & & & \\
\hline R09 & 0.786 & 0.0170 & 0.683 & 0.0317 & & & & & & \\
\hline R10 & 1.598 & 0.0429 & 1.456 & 0.0439 & & & & & & \\
\hline R11 & 0.959 & 0.0056 & 0.930 & 0.0071 & & & & & & \\
\hline R12 & 1.175 & 0.0152 & 1.193 & 0.0181 & & & & & & \\
\hline R13 & 1.347 & 0.0196 & 1.128 & 0.0232 & & & & & & \\
\hline R14 & 0.738 & 0.0199 & 1.130 & 0.0278 & & & & & & \\
\hline R15 & 1.249 & 0.0262 & 1.244 & 0.0289 & & & & & & \\
\hline R16 & 1.487 & 0.0232 & 1.270 & 0.0254 & & & & & & \\
\hline R17 & 0.660 & 0.0217 & 0.720 & 0.0249 & & & & & & \\
\hline R18 & 1.013 & 0.0018 & 1.029 & 0.0024 & & & & & & \\
\hline R19 & 1.451 & 0.0208 & 1.198 & 0.0242 & & & & & & \\
\hline R20 & 1.540 & 0.0819 & 0.989 & 0.1161 & & & & & & \\
\hline
\end{tabular}


Table 8-C. Values of Five-Year Betas and Standard Deviations of the Error Terms

\begin{tabular}{|c|c|c|c|c|c|c|c|c|c|c|}
\hline \multicolumn{11}{|c|}{ Estimates for the five-year intervals covering the years 1990 through 1998} \\
\hline & \multicolumn{2}{|c|}{ 1990-1994 } & \multicolumn{2}{|c|}{ 1991-1995 } & \multicolumn{2}{|c|}{ 1992-1996 } & \multicolumn{2}{|c|}{ 1993-1997 } & \multicolumn{2}{|c|}{ 1994-1998 } \\
\hline Index & Beta & $\operatorname{Std}(\varepsilon)$ & Beta & $\operatorname{Std}(\varepsilon)$ & Beta & $\operatorname{Std}(\varepsilon)$ & Beta & $\operatorname{Std}(\varepsilon)$ & Beta & $\operatorname{Std}(\varepsilon)$ \\
\hline R01 & 0.945 & 0.0115 & 0.960 & 0.0124 & 0.988 & 0.0125 & 1.029 & 0.0118 & 1.005 & 0.0143 \\
\hline R02 & 1.324 & 0.0362 & 1.314 & 0.0342 & 1.065 & 0.0300 & 0.971 & 0.0297 & 0.973 & 0.0318 \\
\hline R03 & 0.534 & 0.0301 & 0.668 & 0.0296 & 0.886 & 0.0294 & 0.652 & 0.0311 & 0.479 & 0.0335 \\
\hline R04 & 1.008 & 0.0056 & 0.977 & 0.0056 & 0.960 & 0.0066 & 0.944 & 0.0068 & 0.993 & 0.0071 \\
\hline R05 & 0.965 & 0.0027 & 0.960 & 0.0029 & 0.945 & 0.0033 & 0.929 & 0.0040 & 0.951 & 0.0052 \\
\hline R06 & 1.218 & 0.0262 & 1.080 & 0.0262 & 1.024 & 0.0287 & 1.052 & 0.0281 & 1.194 & 0.0302 \\
\hline R07 & 1.453 & 0.0304 & 1.286 & 0.0334 & 1.222 & 0.0326 & 1.299 & 0.0357 & 1.326 & 0.0419 \\
\hline R08 & 0.800 & 0.0371 & 0.638 & 0.0308 & 0.542 & 0.0285 & 0.614 & 0.0299 & 0.803 & 0.0322 \\
\hline R09 & 0.962 & 0.0295 & 0.707 & 0.0243 & 0.704 & 0.0256 & 0.627 & 0.0259 & 0.799 & 0.0260 \\
\hline R10 & 1.327 & 0.0368 & 1.133 & 0.0370 & 1.094 & 0.0389 & 1.048 & 0.0400 & 1.280 & 0.0401 \\
\hline R11 & 0.959 & 0.0051 & 0.975 & 0.0055 & 1.010 & 0.0049 & 1.039 & 0.0054 & 1.024 & 0.0064 \\
\hline R12 & 1.141 & 0.0165 & 1.048 & 0.0165 & 0.938 & 0.0176 & 0.885 & 0.0187 & 1.064 & 0.0217 \\
\hline R13 & 1.104 & 0.0293 & 0.961 & 0.0280 & 0.894 & 0.0294 & 0.821 & 0.0287 & 0.988 & 0.0299 \\
\hline R14 & 0.845 & 0.0258 & 0.840 & 0.0261 & 0.813 & 0.0253 & 0.856 & 0.0276 & 1.012 & 0.0365 \\
\hline R15 & 1.035 & 0.0250 & 0.960 & 0.0272 & 0.907 & 0.0275 & 0.894 & 0.0283 & 0.866 & 0.0316 \\
\hline R16 & 1.050 & 0.0371 & 0.972 & 0.0400 & 1.269 & 0.0402 & 1.375 & 0.0443 & 1.318 & 0.0464 \\
\hline R17 & 1.096 & 0.0347 & 1.209 & 0.0336 & 1.187 & 0.0333 & 1.092 & 0.0337 & 0.959 & 0.0289 \\
\hline R18 & 1.008 & 0.0030 & 0.995 & 0.0030 & 0.991 & 0.0032 & 0.984 & 0.0033 & 1.004 & 0.0031 \\
\hline R19 & 1.101 & 0.0282 & 0.935 & 0.0269 & 0.822 & 0.0285 & 0.749 & 0.0277 & 0.952 & 0.0299 \\
\hline R20 & -0.070 & 0.0875 & -0.072 & 0.0862 & 0.445 & 0.0848 & 0.436 & 0.0947 & 1.006 & 0.1091 \\
\hline \multicolumn{11}{|c|}{ Estimates for the five-year intervals covering the years 1995 through 2003} \\
\hline & \multicolumn{2}{|c|}{$1995-1999$} & \multicolumn{2}{|c|}{$1996-2000$} & \multicolumn{2}{|c|}{$1997-2001$} & \multicolumn{2}{|c|}{$1998-2002$} & \multicolumn{2}{|c|}{ 1999-2003 } \\
\hline Index & Beta & $\operatorname{Std}(\varepsilon)$ & Beta & $\operatorname{Std}(\varepsilon)$ & Beta & $\operatorname{Std}(\varepsilon)$ & Beta & $\operatorname{Std}(\varepsilon)$ & Beta & $\operatorname{Std}(\varepsilon)$ \\
\hline R01 & 0.972 & 0.0175 & 0.925 & 0.0201 & 0.926 & 0.0216 & 0.925 & 0.0221 & 0.931 & 0.0202 \\
\hline R02 & 0.911 & 0.0351 & 0.849 & 0.0456 & 0.918 & 0.0506 & 0.847 & 0.0521 & 0.919 & 0.0519 \\
\hline R03 & 0.346 & 0.0357 & 0.164 & 0.0465 & 0.159 & 0.0513 & 0.219 & 0.0577 & 0.284 & 0.0590 \\
\hline R04 & 1.007 & 0.0077 & 0.994 & 0.0121 & 1.007 & 0.0117 & 1.004 & 0.0112 & 0.998 & 0.0110 \\
\hline R05 & 0.930 & 0.0078 & 0.873 & 0.0123 & 0.845 & 0.0132 & 0.836 & 0.0134 & 0.827 & 0.0134 \\
\hline R06 & 1.295 & 0.0407 & 1.385 & 0.0629 & 1.578 & 0.0657 & 1.623 & 0.0640 & 1.653 & 0.0635 \\
\hline R07 & 1.418 & 0.0508 & 1.553 & 0.0661 & 1.802 & 0.0719 & 1.860 & 0.0697 & 1.899 & 0.0663 \\
\hline R08 & 0.788 & 0.0345 & 0.669 & 0.0415 & 0.578 & 0.0437 & 0.470 & 0.0417 & 0.369 & 0.0360 \\
\hline R09 & 0.755 & 0.0362 & 0.672 & 0.0440 & 0.498 & 0.0470 & 0.476 & 0.0476 & 0.367 & 0.0442 \\
\hline R10 & 1.333 & 0.0499 & 1.441 & 0.0710 & 1.720 & 0.0750 & 1.868 & 0.0782 & 1.876 & 0.0786 \\
\hline R11 & 1.038 & 0.0073 & 1.049 & 0.0088 & 1.062 & 0.0092 & 1.067 & 0.0095 & 1.073 & 0.0092 \\
\hline R12 & 1.050 & 0.0255 & 0.968 & 0.0309 & 0.991 & 0.0316 & 0.981 & 0.0314 & 0.909 & 0.0288 \\
\hline R13 & 0.958 & 0.0334 & 0.815 & 0.0444 & 0.832 & 0.0451 & 0.834 & 0.0455 & 0.792 & 0.0435 \\
\hline R14 & 0.988 & 0.0455 & 0.782 & 0.0594 & 0.841 & 0.0652 & 0.671 & 0.0682 & 0.650 & 0.0653 \\
\hline R15 & 0.898 & 0.0427 & 0.875 & 0.0518 & 0.843 & 0.0542 & 0.864 & 0.0561 & 0.989 & 0.0539 \\
\hline R16 & 1.396 & 0.0532 & 1.589 & 0.0608 & 1.798 & 0.0674 & 1.895 & 0.0658 & 1.978 & 0.0628 \\
\hline R17 & 0.908 & 0.0372 & 0.648 & 0.0476 & 0.529 & 0.0508 & 0.489 & 0.0485 & 0.351 & 0.0461 \\
\hline R18 & 1.006 & 0.0033 & 1.003 & 0.0050 & 1.009 & 0.0049 & 1.006 & 0.0047 & 1.001 & 0.0046 \\
\hline R19 & 0.969 & 0.0340 & 0.824 & 0.0485 & 0.857 & 0.0490 & 0.899 & 0.0493 & 0.890 & 0.0480 \\
\hline $\mathrm{R} 20$ & 1.006 & 0.1154 & 0.739 & 0.1156 & 0.554 & 0.1166 & 0.433 & 0.1185 & 0.161 & 0.0933 \\
\hline
\end{tabular}


Table 8-C. (Continued) Values of Five-Year Betas and Standard Deviations of the Error Terms

\begin{tabular}{lcccccccccc}
\hline \multicolumn{8}{c}{ Estimates for the five-year intervals covering the years 2000 through 2008 } \\
\hline Index & Beta & Std $(\varepsilon)$ & Beta & Std $(\varepsilon)$ & Beta & Std $(\varepsilon)$ & Beta & Std $(\varepsilon)$ & Beta & Std $(\varepsilon)$ \\
R01 & 0.927 & 0.0178 & 0.956 & 0.0142 & 0.964 & 0.0109 & 0.975 & 0.0088 & 0.873 & 0.0100 \\
R02 & 0.969 & 0.0499 & 1.051 & 0.0407 & 0.941 & 0.0387 & 1.249 & 0.0354 & 1.020 & 0.0363 \\
R03 & 0.343 & 0.0564 & 0.464 & 0.0476 & 0.570 & 0.0412 & 0.534 & 0.0322 & 0.619 & 0.0326 \\
R04 & 0.994 & 0.0106 & 1.011 & 0.0047 & 0.995 & 0.0046 & 1.048 & 0.0039 & 1.050 & 0.0041 \\
R05 & 0.850 & 0.0122 & 0.874 & 0.0094 & 0.935 & 0.0088 & 1.032 & 0.0077 & 1.080 & 0.0092 \\
R06 & 1.594 & 0.0587 & 1.637 & 0.0324 & 1.389 & 0.0230 & 1.345 & 0.0199 & 1.261 & 0.0188 \\
R07 & 1.831 & 0.0601 & 1.885 & 0.0405 & 1.570 & 0.0287 & 1.356 & 0.0235 & 1.323 & 0.0240 \\
R08 & 0.408 & 0.0326 & 0.431 & 0.0253 & 0.497 & 0.0223 & 0.834 & 0.0220 & 0.590 & 0.0354 \\
R09 & 0.397 & 0.0366 & 0.372 & 0.0274 & 0.546 & 0.0210 & 0.805 & 0.0160 & 0.722 & 0.0263 \\
R10 & 1.820 & 0.0721 & 1.922 & 0.0511 & 1.789 & 0.0497 & 1.469 & 0.0391 & 1.461 & 0.0382 \\
R11 & 1.047 & 0.0085 & 1.035 & 0.0073 & 1.011 & 0.0071 & 0.956 & 0.0057 & 0.916 & 0.0064 \\
R12 & 0.942 & 0.0253 & 0.982 & 0.0198 & 0.951 & 0.0185 & 1.140 & 0.0153 & 1.220 & 0.0153 \\
R13 & 0.846 & 0.0410 & 0.959 & 0.0292 & 0.980 & 0.0282 & 1.308 & 0.0208 & 1.193 & 0.0218 \\
R14 & 0.685 & 0.0602 & 0.735 & 0.0468 & 0.609 & 0.0369 & 1.013 & 0.0284 & 1.063 & 0.0274 \\
R15 & 0.971 & 0.0460 & 1.002 & 0.0354 & 1.110 & 0.0311 & 1.289 & 0.0271 & 1.250 & 0.0295 \\
R16 & 1.962 & 0.0564 & 1.982 & 0.0441 & 1.725 & 0.0325 & 1.371 & 0.0252 & 1.313 & 0.0251 \\
R17 & 0.363 & 0.0401 & 0.476 & 0.0288 & 0.594 & 0.0225 & 0.593 & 0.0231 & 0.703 & 0.0240 \\
R18 & 1.003 & 0.0044 & 1.003 & 0.0022 & 0.993 & 0.0020 & 1.009 & 0.0018 & 1.031 & 0.0021 \\
R19 & 0.923 & 0.0462 & 1.080 & 0.0302 & 1.137 & 0.0286 & 1.437 & 0.0217 & 1.268 & 0.0227 \\
R20 & 0.151 & 0.0880 & 0.237 & 0.0886 & 0.393 & 0.0934 & 1.003 & 0.0824 & 0.972 & 0.1046 \\
\hline
\end{tabular}

Table 8-D. Values of Ten-Year Betas and Standard Deviations of the Error Terms

\begin{tabular}{ccccccccccc}
\hline \multicolumn{8}{c}{ Estimates for the ten-year intervals covering the years 1990 through 2003} \\
\hline Index & Beta & Std $(\varepsilon)$ & Beta & Std $(\varepsilon)$ & Beta & Std $(\varepsilon)$ & Beta & Std $(\varepsilon)$ & Beta & Std $(\varepsilon)$ \\
R01 & 0.956 & 0.0147 & 0.935 & 0.0165 & 0.938 & 0.0176 & 0.949 & 0.0177 & 0.951 & 0.0176 \\
R02 & 1.071 & 0.0364 & 0.976 & 0.0410 & 0.946 & 0.0413 & 0.887 & 0.0422 & 0.926 & 0.0429 \\
R03 & 0.443 & 0.0330 & 0.310 & 0.0399 & 0.297 & 0.0431 & 0.327 & 0.0467 & 0.362 & 0.0478 \\
R04 & 1.004 & 0.0067 & 0.989 & 0.0094 & 0.998 & 0.0095 & 0.989 & 0.0093 & 0.991 & 0.0092 \\
R05 & 0.939 & 0.0060 & 0.897 & 0.0090 & 0.864 & 0.0097 & 0.861 & 0.0100 & 0.872 & 0.0105 \\
R06 & 1.265 & 0.0340 & 1.297 & 0.0481 & 1.473 & 0.0510 & 1.469 & 0.0504 & 1.449 & 0.0506 \\
R07 & 1.451 & 0.0417 & 1.480 & 0.0522 & 1.691 & 0.0561 & 1.701 & 0.0563 & 1.664 & 0.0565 \\
R08 & 0.775 & 0.0357 & 0.654 & 0.0369 & 0.573 & 0.0370 & 0.530 & 0.0368 & 0.540 & 0.0352 \\
R09 & 0.821 & 0.0333 & 0.677 & 0.0356 & 0.538 & 0.0378 & 0.515 & 0.0381 & 0.525 & 0.0373 \\
R10 & 1.353 & 0.0437 & 1.353 & 0.0564 & 1.602 & 0.0601 & 1.672 & 0.0635 & 1.639 & 0.0632 \\
R11 & 1.009 & 0.0066 & 1.029 & 0.0074 & 1.052 & 0.0074 & 1.058 & 0.0077 & 1.053 & 0.0080 \\
R12 & 1.070 & 0.0217 & 0.990 & 0.0246 & 0.980 & 0.0255 & 0.946 & 0.0259 & 0.948 & 0.0260 \\
R13 & 0.999 & 0.0316 & 0.852 & 0.0371 & 0.844 & 0.0378 & 0.828 & 0.0378 & 0.842 & 0.0378 \\
R14 & 0.900 & 0.0370 & 0.794 & 0.0457 & 0.839 & 0.0496 & 0.729 & 0.0518 & 0.784 & 0.0531 \\
R15 & 0.931 & 0.0352 & 0.895 & 0.0413 & 0.857 & 0.0427 & 0.865 & 0.0441 & 0.904 & 0.0447 \\
R16 & 1.270 & 0.0463 & 1.418 & 0.0523 & 1.697 & 0.0556 & 1.758 & 0.0566 & 1.716 & 0.0566 \\
R17 & 0.990 & 0.0358 & 0.810 & 0.0421 & 0.652 & 0.0440 & 0.640 & 0.0429 & 0.621 & 0.0410 \\
R18 & 1.005 & 0.0032 & 1.001 & 0.0041 & 1.005 & 0.0041 & 1.000 & 0.0040 & 1.000 & 0.0039 \\
R19 & 1.000 & 0.0315 & 0.851 & 0.0392 & 0.851 & 0.0398 & 0.863 & 0.0397 & 0.886 & 0.0402 \\
R20 & 0.499 & 0.1037 & 0.502 & 0.1024 & 0.536 & 0.1014 & 0.420 & 0.1064 & 0.438 & 0.1032 \\
\hline
\end{tabular}




\begin{tabular}{ccccccccccc}
\hline \multicolumn{10}{c}{ Estimates for the ten-year intervals covering the years 1995 through 2008 } \\
\hline \multicolumn{1}{c}{ 1995-2004 } & \multicolumn{1}{c}{$1996-2005$} & \multicolumn{2}{c}{$1997-2006$} & \multicolumn{2}{c}{$1998-2007$} & \multicolumn{1}{c}{$1999-2008$} \\
Index & Beta & Std $(\varepsilon)$ & Beta & Std $(\varepsilon)$ & Beta & Std $(\varepsilon)$ & Beta & Std $(\varepsilon)$ & Beta & Std $(\varepsilon)$ \\
R01 & 0.941 & 0.0176 & 0.939 & 0.0172 & 0.938 & 0.0170 & 0.930 & 0.0167 & 0.911 & 0.0160 \\
R02 & 0.905 & 0.0434 & 0.921 & 0.0436 & 0.920 & 0.0449 & 0.926 & 0.0448 & 0.954 & 0.0445 \\
R03 & 0.333 & 0.0468 & 0.313 & 0.0472 & 0.290 & 0.0470 & 0.293 & 0.0473 & 0.402 & 0.0479 \\
R04 & 0.995 & 0.0093 & 0.998 & 0.0092 & 1.002 & 0.0089 & 1.013 & 0.0084 & 1.017 & 0.0083 \\
R05 & 0.870 & 0.0106 & 0.869 & 0.0109 & 0.873 & 0.0114 & 0.874 & 0.0115 & 0.918 & 0.0126 \\
R06 & 1.478 & 0.0505 & 1.496 & 0.0500 & 1.518 & 0.0490 & 1.569 & 0.0473 & 1.513 & 0.0472 \\
R07 & 1.688 & 0.0562 & 1.718 & 0.0550 & 1.731 & 0.0545 & 1.761 & 0.0524 & 1.691 & 0.0509 \\
R08 & 0.545 & 0.0345 & 0.553 & 0.0345 & 0.551 & 0.0344 & 0.528 & 0.0337 & 0.453 & 0.0363 \\
R09 & 0.521 & 0.0372 & 0.523 & 0.0371 & 0.512 & 0.0361 & 0.531 & 0.0356 & 0.496 & 0.0368 \\
R10 & 1.666 & 0.0630 & 1.673 & 0.0623 & 1.741 & 0.0631 & 1.810 & 0.0618 & 1.723 & 0.0621 \\
R11 & 1.053 & 0.0081 & 1.048 & 0.0082 & 1.046 & 0.0083 & 1.044 & 0.0081 & 1.017 & 0.0086 \\
R12 & 0.952 & 0.0260 & 0.966 & 0.0258 & 0.979 & 0.0257 & 1.004 & 0.0246 & 1.023 & 0.0239 \\
R13 & 0.847 & 0.0380 & 0.865 & 0.0377 & 0.877 & 0.0375 & 0.916 & 0.0359 & 0.939 & 0.0352 \\
R14 & 0.783 & 0.0536 & 0.752 & 0.0531 & 0.760 & 0.0531 & 0.738 & 0.0522 & 0.799 & 0.0504 \\
R15 & 0.895 & 0.0447 & 0.906 & 0.0448 & 0.923 & 0.0445 & 0.943 & 0.0443 & 1.085 & 0.0435 \\
R16 & 1.747 & 0.0559 & 1.774 & 0.0534 & 1.777 & 0.0526 & 1.793 & 0.0504 & 1.739 & 0.0494 \\
R17 & 0.611 & 0.0402 & 0.593 & 0.0398 & 0.556 & 0.0394 & 0.501 & 0.0377 & 0.478 & 0.0372 \\
R18 & 1.002 & 0.0039 & 1.002 & 0.0038 & 1.003 & 0.0037 & 1.007 & 0.0036 & 1.012 & 0.0036 \\
R19 & 0.903 & 0.0408 & 0.923 & 0.0408 & 0.944 & 0.0403 & 0.996 & 0.0387 & 1.028 & 0.0382 \\
R20 & 0.448 & 0.1040 & 0.449 & 0.1041 & 0.485 & 0.1058 & 0.538 & 0.1016 & 0.454 & 0.0997 \\
\hline
\end{tabular}

\section{Estimation and Testing of Gammas}

The regression equation to estimate the gamma coefficients is:

$$
R_{p t}=\hat{\gamma}_{0 t}+\hat{\gamma}_{1 t} \hat{\beta}_{p, t-1}+\hat{\gamma}_{2 t} \hat{\beta}_{p, t-1}^{2}+\hat{\gamma}_{3 t} \bar{s}_{p, t-1}\left(\hat{\varepsilon}_{i}\right)+\hat{\eta}_{p t}
$$

To find the gammas, we need the values of betas and the standard deviation of the error term $\varepsilon_{i t}$. In Fama and MacBeth (1973), the values of gammas are determined using betas calculated from the monthly returns of periods spanning five to eight years. The returns on many of the mutual funds are reported for periods of one year, three years, five years, and ten years in the financial press. In a similar fashion, in the current study, for purposes of estimating the gammas, the betas are calculated using monthly returns of four different time intervals as described below:

(a) One-year interval: The betas calculated using the 12 monthly returns of the previous year are used to estimate the gammas for the 12 months of the current year. For example, betas calculated using the monthly returns of the year 1990 are used in estimating the gammas for the 12 months of the year 1991. In other words, we find the betas for the each of years 1990,1991,.., 2008, and use them to estimate the gammas for each of the years 1991, 1992, ..., 2009 respectively.

(b) Three-year interval: In this case, we use returns of 36 months from the previous three years to find the betas, and use the betas to estimate the gammas for the 12 months of the current year. For example, we find the betas using the monthly returns of the years 1990, 1991, and 1992; then, we use the betas to estimate the gammas for the 12 months of the year 1993. Then, on a rolling basis, the betas determined using the monthly returns of the years 1991, 1992 and 1993 are used to estimate the gammas for the 12 months of the year 1994, and so on until 2009.

(c) Five-year interval: The procedure is the same as in the three-year case. Now, we use returns of 60 months from the previous five years to find the betas, and use the betas to estimate the gammas for the 12 months of the current year. For example, we find the betas using the monthly returns of the years 1990, 1991, 1992, 1993, and 1994; then, we use the betas to estimate the gammas for the 12 months of the year 1995. Then, on a rolling basis, the betas determined using the monthly returns of the years 1991, 1992, 1993, 1994, and 1995 are used to estimate the gammas for the 12 months of the year 1996, and so on until 2009.

(d) Ten-year interval: Monthly returns of the past ten years are used to determine the betas, which are then used to estimate the gammas for the 12 months of the current year. For example, betas based on the 120 monthly returns of the ten years spanning the interval of 1990 through 1999 are used to estimate the gammas for the 12 months of the year 2000. Then, on a rolling basis, betas are determined using the monthly returns of the period 1991-2000; the betas are then used in estimating the gammas for the 12 months of the year 2001, and so on until 2009. 
For purposes of empirical testing, equation (3) can be split into four equations as given below:

$$
\begin{aligned}
& R_{p t}=\hat{\gamma}_{0 t}+\hat{\gamma}_{1 t} \hat{\beta}_{p, t-1}+\hat{\eta}_{p t} \\
& R_{p t}=\hat{\gamma}_{0 t}+\hat{\gamma}_{1 t} \hat{\beta}_{p, t-1}+\hat{\gamma}_{2 t} \hat{\beta}_{p, t-1}^{2}+\hat{\eta}_{p t} \\
& R_{p t}=\hat{\gamma}_{0 t}+\hat{\gamma}_{1 t} \hat{\beta}_{p, t-1}+\hat{\gamma}_{3 t} \bar{s}_{p, t-1}\left(\hat{\varepsilon}_{i}\right)+\hat{\eta}_{p t} \\
& R_{p t}=\hat{\gamma}_{0 t}+\hat{\gamma}_{1 t} \hat{\beta}_{p, t-1}+\hat{\gamma}_{2 t} \hat{\beta}_{p, t-1}^{2}+\hat{\gamma}_{3 t} \bar{s}_{p, t-1}\left(\hat{\varepsilon}_{i}\right)+\hat{\eta}_{p t}
\end{aligned}
$$

The basic assumption of the CAPM is that the return on any asset is a linear function of its beta. It implies that $\gamma_{1}$ should be strictly positive. Further, $\gamma_{2}$ should be zero. If $\gamma_{2}$ were positive or negative, it implies that the relation between the asset return and its beta is nonlinear, which is contrary to the CAPM. The coefficient $\gamma_{3}$ is associated with the idiosyncratic risk or non-beta risk, which cannot be diversified. Since it cannot be diversified, the value of the $\gamma_{3}$ should also be zero. It cannot be positive or negative.

The conditions to be tested empirically to validate the CAPM are that (1) $\gamma_{1}>0$, (2) $\gamma_{2}=0$, (3) $\gamma_{3}=0$, and (4) $\gamma_{0}>0$ or $\gamma_{0}=R_{f}$, where $R_{f}$ is the risk-free rate. In case of the time intervals involving one year, three years or five-years, we fail to reject the null hypothesis of $\gamma_{j}=0, j=0,1,2,3$ in all the panels (at $5 \%$ level of significance). In case of the ten-year interval, in Panel $\mathrm{A}, \gamma_{0}>0$ at $5 \%$ level of significance, but $\gamma_{1}$ has a negative sign, the opposite of what is expected. In Panel C also, the situation is the same (at 10\% level of significance). In Panel D, the parameter $\gamma_{1}$ has a positive sign and is significant at 5\% level, but $\gamma_{2}$ and $\gamma_{3}$ which should be equal to zero, turn out to be different from zero at $5 \%$ level of significance. None of the conditions that validate CAPM are satisfied. The empirical evidence of this study does not support the CAPM. Roll (1977) has stated that the CAPM cannot be tested or validated since the market portfolio cannot be identified. However, Pasquariello (1999) has tested the CAPM using the original data of Fama and Macbeth (1973) with some improvements in the estimation procedures and found evidence in support of the CAPM. It is not clear whether the approach of Pasquariello (1999) would yield any favorable results in the current study.

The values of the gamma coefficients obtained from equations (3a), (3b), (3c) and (3d) are given in Panels A, B, C,

\begin{tabular}{|c|c|c|c|c|c|c|c|c|}
\hline \multirow{2}{*}{$\begin{array}{l}\text { Beta } \\
\text { Estimation } \\
\text { Interval }\end{array}$} & \multicolumn{2}{|c|}{$\gamma_{0}$} & \multicolumn{2}{|c|}{$\gamma_{1}$} & \multicolumn{2}{|c|}{$\gamma_{2}$} & \multicolumn{2}{|c|}{$\gamma_{3}$} \\
\hline & $\begin{array}{c}\text { Mean } \\
\text { (St. Dev.) }\end{array}$ & $\begin{array}{c}\text { Ttstat } \\
\text { (p-vlaue) }\end{array}$ & $\begin{array}{l}\text { Mean } \\
\text { (St. Dev.) }\end{array}$ & $\begin{array}{c}\text { t-stat } \\
\text { (p-vlaue) }\end{array}$ & $\begin{array}{c}\text { Mean } \\
\text { (St. Dev.) }\end{array}$ & $\begin{array}{c}\text { t-stat } \\
\text { (p-vlaue) }\end{array}$ & $\begin{array}{c}\text { Mean } \\
\text { (St. Dev.) }\end{array}$ & $\begin{array}{c}\text { t-stat } \\
\text { (p-vlaue) }\end{array}$ \\
\hline \multicolumn{9}{|c|}{ Panel A: $R_{p t}=\hat{\gamma}_{0 t}+\hat{\gamma}_{1 t} \hat{\beta}_{p, t-1}+\hat{\eta}_{p t}$} \\
\hline One-year & $\begin{array}{c}0.004 \\
(0.053)\end{array}$ & $\begin{array}{c}1.257 \\
(0.105)\end{array}$ & $\begin{array}{l}0.003 \\
(0.061)\end{array}$ & $\begin{array}{c}0.853 \\
(0.197)\end{array}$ & & & & \\
\hline Three-year & $\begin{array}{c}0.001 \\
(0.076)\end{array}$ & $\begin{array}{c}0.220 \\
(0.413)\end{array}$ & $\begin{array}{c}0.007 \\
((0.083)\end{array}$ & $\begin{array}{l}1.129 \\
(0.130)\end{array}$ & & & & \\
\hline Five-year & $\begin{array}{l}-0.001 \\
(0.086)\end{array}$ & $\begin{array}{l}-0.128 \\
(0.449)\end{array}$ & $\begin{array}{c}0.009 \\
(0.097)\end{array}$ & $\begin{array}{l}1.246 \\
(0.07)\end{array}$ & & & & \\
\hline Ten-year & $\begin{array}{c}0.011 \\
(0.074)\end{array}$ & $\begin{array}{c}1.671 \\
(0.049)\end{array}$ & $\begin{array}{l}-0.010 \\
(0.088)\end{array}$ & $\begin{array}{l}-1.193 \\
(0.118)\end{array}$ & & & & \\
\hline \multicolumn{9}{|c|}{ Panel B: $R_{p t}=\hat{\gamma}_{0 t}+\hat{\gamma}_{1 t} \hat{\beta}_{p, t-1}+\hat{\gamma}_{2 t} \hat{\beta}_{p, t-1}^{2}+\hat{\eta}_{p t}$} \\
\hline One-year & $\begin{array}{c}0.000 \\
(0.094)\end{array}$ & $\begin{array}{c}0.006 \\
(0.498)\end{array}$ & $\begin{array}{c}0.015 \\
(0.198)\end{array}$ & $\begin{array}{c}1.141 \\
(0.127)\end{array}$ & $\begin{array}{l}-0.007 \\
(0.108)\end{array}$ & $\begin{array}{l}-0.940 \\
(0.174)\end{array}$ & & \\
\hline Three-year & $\begin{array}{l}0.001 \\
(0.107)\end{array}$ & $\begin{array}{c}0.186 \\
(0.426)\end{array}$ & $\begin{array}{c}0.005 \\
(0.220)\end{array}$ & $\begin{array}{c}0.355 \\
(0.362)\end{array}$ & $\begin{array}{l}0.001 \\
(0.126)\end{array}$ & $\begin{array}{l}0.097 \\
(0.462)\end{array}$ & & \\
\hline Five-year & $\begin{array}{c}0.003 \\
(0.135)\end{array}$ & $\begin{array}{c}0.279 \\
(0.390)\end{array}$ & $\begin{array}{c}0.002 \\
(0.284)\end{array}$ & $\begin{array}{l}0.103 \\
(0.459)\end{array}$ & $\begin{array}{l}0.003 \\
(0.162)\end{array}$ & $\begin{array}{l}0.283 \\
(0.389)\end{array}$ & & \\
\hline Ten-year & $\begin{array}{c}0.001 \\
(0.079)\end{array}$ & $\begin{array}{c}0.188 \\
(0.426)\end{array}$ & $\begin{array}{c}0.014 \\
(0.179)\end{array}$ & $\begin{array}{c}0.830 \\
(0.204)\end{array}$ & $\begin{array}{l}-0.013 \\
(0.111)\end{array}$ & $\begin{array}{l}-1.259 \\
(0.105)\end{array}$ & & \\
\hline
\end{tabular}
and $\mathrm{D}$ of Table 9 . For each of the four cases described, the betas and standard deviations of the error term $\varepsilon$ are given Tables 8-A, 8-B, 8-C and 8-D.

Table 9. Estimated Values of Gammas 


\begin{tabular}{lcccccccc}
\hline & \multicolumn{7}{c}{ Panel C: $R_{p t}=\hat{\gamma}_{0 t}+\hat{\gamma}_{1 t} \hat{\beta}_{p, t-1}+\hat{\gamma}_{3 t} \bar{s}_{p, t-1}\left(\hat{\varepsilon}_{i}\right)+\hat{\eta}_{p t}$} \\
One-year & 0.004 & 1.004 & 0.004 & 1.082 & & 0.010 & 0.190 \\
& $(0.055)$ & $(0.158)$ & $(0.060)$ & $(0.140)$ & & $(0.829)$ & $(0.425)$ \\
Three-year & 0.000 & 0.087 & 0.006 & 1.028 & & 0.045 & 0.755 \\
& $(0.071)$ & $(0.465)$ & $(0.082)$ & $(0.153)$ & & $(0.854)$ & $(0.225)$ \\
Five-year & -0.003 & -0.420 & 0.009 & 1.215 & & 0.056 & 0.871 \\
& $(0.081)$ & $(0.337)$ & $(0.096)$ & $(0.113)$ & & $(0.865)$ & $(0.192)$ \\
Ten-year & 0.010 & 1.308 & -0.011 & -1.285 & & & 0.059 & 0.912 \\
& $(0.083)$ & $(0.097)$ & $(0.092)$ & $(0.101)$ & & & $(0.713)$ & $(0.182)$ \\
& Panel D: & $R_{p t}=\hat{\gamma}_{0 t}+\hat{\gamma}_{1 t} \hat{\beta}_{p, t-1}+\hat{\gamma}_{2 t} \hat{\beta}_{p, t-1}^{2}+\hat{\gamma}_{3 t} \bar{s}_{p, t-1}\left(\hat{\varepsilon}_{i}\right)+\hat{\eta}_{p t}$ & \\
One-year & -0.003 & -0.314 & 0.018 & 1.096 & -0.008 & -0.888 & 0.042 & 0.681 \\
& $(0.126)$ & $(0.377)$ & $(0.249)$ & $(0.137)$ & $(0.135)$ & $(0.188)$ & $(0.927)$ & $(0.248)$ \\
Three-year & 0.002 & 0.284 & -0.001 & -0.052 & 0.005 & 0.441 & 0.074 & 1.094 \\
& $(0.123)$ & $(0.388)$ & $(0.250)$ & $(0.479)$ & $(0.148)$ & $(0.330)$ & $(0.962)$ & $(0.138)$ \\
Five-year & 0.000 & -0.033 & 0.002 & 0.103 & 0.004 & 0.370 & 0.079 & 1.140 \\
& $(0.127)$ & $(0.487)$ & $(0.267)$ & $(0.459)$ & $(0.161)$ & $(0.356)$ & $(0.935)$ & $(0.128)$ \\
Ten-year & -0.012 & -1.294 & 0.032 & 1.678 & -0.021 & -1.884 & 0.133 & 1.652 \\
& $(0.103)$ & $(0.099)$ & $(0.210)$ & $(0.048)$ & $(0.124)$ & $(0.031)$ & $(0.885)$ & $(0.051)$ \\
\hline
\end{tabular}

\section{Conclusions}

In this paper, we do an analysis of the monthly returns for twenty different stock indices that represent a broad sample of the market for the twenty year time period from 1990 to 2009. We compute Fama-MacBeth betas for each portfolio for one-year, two-year, four-year, five-year, ten-year and twenty-year time periods. We perform unit root tests on the one-year interval betas and find that only the Gold and Silver Index is stationary. We perform co-integration tests on five quintiles based on high to low betas for the twenty indices and find that the middle quintile with betas close to one, is not co-integrated while the other four quintiles are co-integrated.

Our empirical results indicate that the Gold and Silver Index has the highest volatility. The S\&P Healthcare Composite Index exhibits a level of volatility comparable to that of the Dow Jones Utilities Index, but its average yield is double that of the Utilities Index, making it a safer investment vehicle. The time-series of the betas of all the indices except that of Gold and Silver Index calculated from the monthly returns of one-year intervals are found to be non-stationary. However, the betas in four of the five quintiles are found to be co-integrated.

We perform gamma analysis on the one-year, two-year, four-year, five-year, ten-year interval betas to determine if the betas are consistent with the CAPM. That is, we test to determine if the returns for the indices are a linear function of the index betas. The results of the empirical tests on the gamma coefficients do not support the CAPM.

In a nutshell, we use the Fama-MacBeth regression analysis methodology to determine if twenty indices for the twenty year time period from 1990 to 2009 provide a linear relationship between the index returns and index betas. We find that the empirical results are not consistent with the assumptions of the CAPM. For our time period and our sample of indices, the empirical results based on the gamma tests of the relationship between index returns and index betas do not support the CAPM.

\section{References}

Fama, Eugene F., \& James D. MacBeth. (1973). Risk, Return, and Equilibrium: Empirical Tests, Journal of Political Economy, Vol. 81, No.3, pp 607-636. http://dx.doi.org/10.1086/260061

Fama, Eugene F., \& Kenneth R. French. (1992). The Cross-section of Expected Stock Returns, Journal of Finance, Vol. 47, No.2, pp 427-465. http://dx.doi.org/10.1111/j.1540-6261.1992.tb04398.x

Fama, Eugene F., \& Kenneth R. French. (1996). Multifactor Explanations of Asset Pricing Anomalies, Journal of Finance, Vol. 51, No.1, pp 55-84. http://dx.doi.org/10.1111/j.1540-6261.1996.tb05202.x

Jagannathan, Ravi, \& Zhenyu Wang. (1996). The Conditional CAPM and the Cross-section Expected Stock Returns, Journal of Finance, Vol. 51, No.1, pp 3-53. http://dx.doi.org/10.1111/j.1540-6261.1996.tb05201.x

Pasquariello, Paolo. (1999). The Fama-Macbeth Approach Revisited, Working Paper, University of Michigan, Ann Arbor, Michigan.

Roll, Richard. (1977). A Critique of the Asset Pricing Theory's Tests, Part I: On the Past and Potential Testability of the Theory, Journal of Financial Economics, Vol. 4, pp. 129-176. http://dx.doi.org/10.1016/0304-405X(77)90009-5 